\title{
1 Genomic landscape of lung adenocarcinoma in East Asians
}

2 Jianbin Chen ${ }^{1}$, Hechuan Yang ${ }^{1,2}$, Audrey Su Min Teo ${ }^{1}$, Lidyana Bte Amer ${ }^{1}$, Faranak Ghazi 3 Sherbaf $^{1}$, Chu Quan Tan ${ }^{1}$, Jacob Josiah Santiago Alvarez ${ }^{1}$, Bingxin Lu ${ }^{1}$, Jia Qi Lim ${ }^{1}$, Angela 4 Takano $^{3}$, Rahul Nahar ${ }^{1}$, Yin Yeng Lee ${ }^{1}$, Cheryl Zi Jin Phua ${ }^{1}$, Khi Pin Chua ${ }^{1}$, Lisda Suteja ${ }^{4}$, 5 Pauline Jieqi Chen ${ }^{1}$, Mei Mei Chang ${ }^{1}$, Tina Puay Theng Koh ${ }^{5}$, Boon-Hean $\mathrm{Ong}^{6}$, Devanand 6 Anantham $^{7}$, Anne Ann Ling Hsu ${ }^{7}$, Apoorva Gogna ${ }^{8}$, Chow Wei Too ${ }^{8}$, Zaw Win Aung 9 , Yi

7 Fei Lee ${ }^{1,13}$, Lanying Wang ${ }^{9}$, Tony Kiat Hon Lim $^{3}$, Andreas Wilm ${ }^{1}$, Poh Sum Choi ${ }^{1}$, Poh 8 Yong $\mathrm{Ng}^{1}$, Chee Keong $\mathrm{Toh}^{4}$, Wan-Teck Lim ${ }^{4,14}$, Siming $\mathrm{Ma}^{1}$, Bing Lim ${ }^{1}$, Jin Liu ${ }^{10}$, Wai 9 Leong Tam ${ }^{1,11,12,13}$, Anders Jacobsen Skanderup ${ }^{1}$, Joe Poh Sheng Yeong ${ }^{3,14}$, Eng-Huat Tan ${ }^{9,4}$, 10 Caretha L. Creasy ${ }^{15}$, Daniel Shao Weng $\operatorname{Tan}^{1,4,16, *}$, Axel M. Hillmer ${ }^{1,17, *}$, Weiwei 11 Zhai ${ }^{1,2,13,18, *}$

\section{Affiliations}

$13{ }^{1}$ Genome Institute of Singapore, Agency for Science, Technology and Research (A*STAR), 14 Singapore 138672, Singapore

$15{ }^{2}$ Key Laboratory of Zoological Systematics and Evolution, Institute of Zoology, Chinese

16 Academy of Sciences, Beijing 100101, China

$17{ }^{3}$ Department of Anatomical Pathology, Singapore General Hospital, Singapore 169608,

18 Singapore.

$19{ }^{4}$ Division of Medical Oncology, National Cancer Centre Singapore, Singapore 169610, 20 Singapore.

$21{ }^{5}$ Surgical Oncology, National Cancer Centre Singapore, Singapore 169610, Singapore.

${ }^{6}$ Department of Cardiothoracic Surgery, National Heart Centre Singapore, Singapore 169609, 23 Singapore.

$24{ }^{7}$ Department of Respiratory \& Critical Care Medicine, Singapore General Hospital, 25 Singapore 169854, Singapore.

$26{ }^{8}$ Department of Vascular \& Interventional Radiology, Singapore General Hospital, Singapore 27 169609, Singapore.

$28{ }^{9}$ Division of Clinical Trials and Epidemiological Sciences, National Cancer Centre Singapore, 29 Singapore 169610, Singapore.

$30{ }^{10}$ Centre for Quantitative Medicine, Program in Health Services and Systems Research, 31 Duke-NUS Medical School, Singapore 169857, Singapore.

$32{ }^{11}$ Cancer Science Institute of Singapore, National University of Singapore, 14 Medical Drive, 33 Singapore 117599, Singapore

$34{ }^{12}$ Department of Biochemistry, Yong Loo Lin School of Medicine, National University of 35 Singapore, 10 Medical Drive, Singapore 117597, Singapore

$36{ }^{13}$ School of Biological Sciences, Nanyang Technological University, 60 Nanyang Drive,

37 Singapore 637551, Singapore

$38{ }^{14}$ Institute of Molecular and Cell Biology, Singapore 138673, Singapore.

$39{ }^{15}$ Oncology R\&D, GlaxoSmithKline, Collegeville, PA 19426-0989, United States. 
$40{ }^{16}$ Cancer Therapeutics Research Laboratory, Division of Medical Sciences, National Cancer 41 Centre Singapore, Singapore 169610, Singapore.

$42{ }^{17}$ Institute of Pathology, Faculty of Medicine and University Hospital Cologne, University of 43 Cologne, 50937 Cologne, Germany

$44{ }^{18}$ Center for Excellence in Animal Evolution and Genetics, Chinese Academy of Sciences, 45 Kunming 650223, China

46 * jointly supervised this work. Email: daniel.tan.s.w@nccs.com.sg; ahillmer@uni-koeln.de ; 47 weiweizhai@ioz.ac.cn 


\section{Abstract}

49 Lung cancer is the world's leading cause of cancer death with strong ancestry disparities. By 50 sequencing and assembling the largest genomic and transcriptomic dataset of lung 51 adenocarcinoma (LUAD) in individuals of East Asian ancestry (EAS; $n=305$ ) to date, we 52 found that East Asian LUADs had more stable genomes characterized by fewer mutations 53 and less copy number alteration than LUADs from individuals of European ancestry (EUR). 54 This difference is much stronger in smokers as compared to non-smokers. Transcriptomic 55 clustering identified a novel EAS-specific LUAD subgroup with a less complex genomic 56 profile and up-regulated immune-related genes, allowing the possibility of immunotherapy57 based approaches. Integrative analysis across clinical and molecular features showed the 58 importance of molecular phenotypes in patient prognostic stratification. EAS LUADs had 59 better prediction accuracy than those of European ancestry, potentially due to the less 60 complex genomic architecture. This study elucidated a comprehensive genomic landscape of 61 EAS LUADs and highlighted important ancestry differences between the two cohorts. 
64 Lung adenocarcinoma (LUAD) is the most common type of lung cancer with over one 65 million deaths annually world-wide ${ }^{1,2}$. Several large-scale genomic studies have analyzed the 66 genomic landscape of $\operatorname{LUAD}^{3-6}$ and characterized important therapeutic targets such as 67 EGFR and ALK. Moreover, immune checkpoint blockade (ICB) has been associated with 68 significant efficacy in LUAD patients with high PDL1 (CD274) expression, tumor mutation 69 burden (TMB) and gene expression profile (GEP) score ${ }^{7-11}$. Despite this progress, how to 70 stratify patients for treatment with appropriate therapeutic approaches remains a challenge of 71 high priority ${ }^{12}$.

72 Genomic studies of LUAD thus far has been based on smoker-dominated populations of 73 European ancestry. However, LUAD seems to differ markedly between individuals of Asian74 and those of European-ancestry. For example, EAS LUADs are enriched for female non75 smokers, while most EUR LUADs tend to occur in male smokers. Mutations in EGFR are 76 present in $40-60 \%$ of EAS LUADs but only in $7-10 \%$ of EUR LUADs ${ }^{13-17}$. Moreover, 77 several novel driver genes in EAS have been discovered in modestly sized Chinese cohorts ${ }^{17-}$ $78^{19}$, suggesting that genomic disparities may be larger than previously expected. Intra-tumor heterogeneity (ITH), an important metric for tumor evolution and patient survival, was also 80 reported to be higher in EAS EGFR-mutant non-smokers than in the EUR counterparts ${ }^{20}$. 81 Despite these observations, the ancestry differences in LUAD genomics have yet to be 82 elucidated systematically, due to the lack of a sufficiently large EAS cohort.

83 To address the above challenges, we sequenced the whole exomes $(n=210)$ and 84 transcriptomes $(n=181)$ of 213 Chinese LUAD patients from Singapore. Along with the 85 previously published whole exome sequencing (WES) data of 92 Chinese patients ${ }^{17}$, we 86 present here the largest genomic and transcriptomic dataset for EAS LUAD $(n=305)$ to date. 87 By comparing the genomic landscapes of LUAD from EAS and EUR ${ }^{4}$ using the same 88 analysis pipelines, we identified strong differences in driver mutations, copy number 89 alterations (CNAs), and RNA transcriptomic subgroups. Integrating a network of clinical and 90 genomic features, we found EAS LUADs have better prediction accuracy for patient 91 outcomes than EUR LUADs, possibly due to the more stable genomic profiles of EAS 92 LUADs. We depicted a comprehensive genomic landscape of EAS LUADs and characterized 93 the complex ancestry differences with important clinical implications.

\section{Results}




\section{Patient samples and sequencing}

96 We recruited 213 LUAD patients of Chinese descent from Singapore and performed WES 97 and transcriptome sequencing on 210 and 181 patients, respectively (Supplementary Table 1).

98 The mean coverage for the WES was $89 \mathrm{X}(51.5-182.1 \mathrm{X})$ and the average number of paired99 end reads for the RNA sequencing was 31.1M (11.9-88.7M). We included WES data of 92 100 Chinese patients from a recent study by Beijing Genome Institute (BGI) ${ }^{17}$ to form the largest 101 EAS LUAD cohort (the "EAS cohort", $\mathrm{n}=305$, Fig. 1a, Supplementary Table 1, 102 Supplementary Fig. 1). To assess the ancestry differences, we also downloaded and processed 103 raw exome and RNA sequencing data for 249 LUAD patients of European ancestry from The 104 Cancer Genome Atlas (TCGA) using the same analysis procedures ${ }^{21-25}$ (the "EUR cohort", 105 Fig. 1a, Supplementary Table 2, Supplementary Fig. 2, Supplementary Note). Compared to 106 the EAS cohort, the EUR patients included more smokers with higher cigarette pack-years 107 (Fig. 1b, Supplementary Fig. 1). In general, the two cohorts were similar in stage and age as 108 well as other clinical features. Despite the limited number of EUR never-smokers $(\mathrm{n}=68)$, our 109 EAS cohort allowed for direct comparison between smokers and never smokers across two 110 ancestry backgrounds.

111 Using Mutect $^{26}$ and Strelka ${ }^{27}, 67,274$ single nucleotide variants (SNVs) and 6,404 112 insertions/deletions (indels) were discovered in the combined EAS cohort (Supplementary 113 Table 3). With target amplicon sequencing (Methods), we successfully validated 325 variants 114 out of 335 SNVs (true positive rate of $97 \%$ after additional filtering of variants, Methods, 115 Supplementary Note, Supplementary Table 4). The median TMB of our EAS cohort was 2.04 116 per $\mathrm{Mb}$ (mean 4.05), much lower than that of the EUR cohort (median 5.08, mean 7.78).

117 Using a multivariate linear model, we found smoking status to be the major variable 118 correlating with TMB (Supplementary Fig. 3), where the TMBs of non-smokers (EAS: 119 median 1.81/Mb, mean 2.31/Mb; EUR: median $1.81 / \mathrm{Mb}$, mean 3.17/Mb) were much lower 120 than the TMBs of smokers (EAS: median 3.29/Mb, mean 6.96/Mb; EUR: median 7.01/Mb, 121 mean 9.66/Mb) in both cohorts. Notably, both EAS smokers and non-smokers show

122 significantly lower mutation burden than their EUR counterparts (Fig 1b, q-value=0.04 for 123 smokers, 0.07 for non-smokers) even after controlling for possible confounding factors 124 including the subtle difference in the reference genome (Supplementary Fig. 3,4; 125 Supplementary Note).

\section{Driver genes in EAS LUADs}


127 With larger sample sizes, we aimed to identify driver genes, especially those median to rare 128 drivers in the EAS cohort. By combining two methods (MutSigCV ${ }^{28}$ and 20/20+ ${ }^{29}$ ), we 129 identified 27 drivers at a false discovery rate of 0.1 (Fig. 1c, Supplementary Table 5, 130 Supplementary Fig. 5, 6). The most frequent drivers were EGFR (47\%), TP53 (36\%), and 131 KRAS (11\%). Potential fusion driver events were also detected ${ }^{30}$ and validated using 132 transcriptomic data from the Singapore samples (Fig. 1c, Methods, Supplementary Table 6).

133 Low driver abundance seemed to be a dominant characteristic of the EAS LUADs, 134 concordant with the lower TMB profile. The average number of LUAD drivers per patient 135 was only 2.08 among EAS non-smokers (2.65 among EUR nonsmokers, p-value=0.045) and 1363.64 among EAS smokers (5.56 among EUR smokers, p-value $=4.2 \times 10^{-6}$ ), while $13.24 \%$ of 137 all the patients had no known driver mutations or driver fusion events $(2.81 \% \text { in EUR })^{31,32}$.

138 In addition to the known driver mutations, we also identified seven novel LUAD driver genes: 139 PARP4 (6\%), EPRS (4\%), LYST (4\%), NCOR2 (2\%), PBRM1 (2\%), RASA1 (2\%), and 140 ZMYM2 (2\%). NCOR2 and PBRM1 are listed in the Cancer Gene Census for other cancer 141 types. NCOR2 is known to drive tumorigenesis in prostate cancer ${ }^{33}$, while PBRM1 is 142 described as a tumor suppressor gene for both renal and breast cancer ${ }^{34}$. PARP4 codes for a 143 member of the PARP protein superfamily regulating DNA repair and genome instability, and 144 is related to various cancer types ${ }^{35-37}$. The other novel drivers such as EPRS ${ }^{38}, \operatorname{LYST}^{39}$, $145 R_{A S A} 1^{40,41}$ and $Z M Y M 2^{42}$ showed mutational patterns typical of tumor suppressors 146 (Supplementary Fig. 5), and were known to be involved in tumorigenesis in other cancer 147 types.

148 When comparing driver genes across the cohorts, 15 genes among smokers and 4 among non149 smokers showed significant differences in frequencies (Fig. 1d). The EAS cohort harbored 150 more patients with mutations in EGFR and PARP4, but had lower frequencies for classical 151 driver genes such as KEAP1, STK11, NF1, BRAF, and KRAS. Most of such differences 152 remained significant even after controlling for other confounding factors or restricting to only 153 functional mutations ${ }^{43}$ (Supplementary Fig. 7, 8). Overall, we demonstrated that driver 154 composition among EAS and EUR LUAD patients were different.

\section{Correlation between drivers and clinical phenotypes}

156 Correlating driver mutations with clinical features in the EAS cohort, we found that 157 mutations in EGFR were enriched in females, while TP53, KRAS, APC, EPRS, LYST, and 158 KEAP1 mutations were enriched in males and smokers (Fig. 1e). TP53 mutations occurred 
159 more frequently in younger patients (mean age 61.3 vs 64.0, q-value $=0.098$, Methods), 160 whereas RBM10 mutations occurred more in older patients (mean age 72.1 vs 62.3, q161 value $=0.057)$. Subsequently, we tested possible correlations between driver status and patient 162 survival using multivariate Cox models to account for clinical variables (stage, smoking, 163 gender, and age). In the EAS cohort, mutations in four drivers (KEAP1, PBRM1, APC, and 164 PDGFRA) correlated significantly with poor survival, but mutations in EGFR conferred 165 survival benefit ${ }^{44-46}$ (Extended Data Fig. 1, Supplementary Fig. 9). Applying the same 166 analytical pipeline to the EUR cohort, a different set of drivers (CDC27, STK11, SETBP1, 167 and SKIV2L2) were found to stratify the patients (Extended Data Fig. 1). In terms of driver 168 co-occurrence and mutual exclusivity, mutations in TP53, NAV3, LRP1B, and several other 169 genes often co-occurred in the EAS cohort, whereas the only mutually exclusive driver pair 170 was EGFR and KRAS (Supplementary Fig. 10). This corroborates the notion that EGFR is a 171 dominant driver and tends to drive tumorigenesis alone ${ }^{20}$.

172 Ancestry differences in copy number alterations

173 We identified significantly altered copy number variations (CNV) using Sequenza ${ }^{47}$ and 174 GISTIC 2.0 algorithm $^{48}$. At the chromosomal level, EAS cohort showed a lower amount of 175 arm-level CNV than the EUR cohort, and such differences were more pronounced in 176 deletions (Fig. 2a, Supplementary Table 7). However, many focal CNVs around driver-gene 177 amplifications in EGFR, MYC, and KRAS, as well as deletions in FAT1, APC, and STK11, 178 were found in both cohorts (Fig. 2b, Supplementary Table 8, Supplementary Fig. 11-12).

179 We further inferred the CNVs for both the smoker and non-smokers across the two cohorts 180 and calculated ploidy, genome doubling, and percentage of genome altered (i.e. genomic instability index or GII) (Methods). Compared to the EUR smokers, the EAS smokers exhibited significantly lower levels of aneuploidy, had a lower percentage of tumors with genome doubling, and showed lower GII, which was mainly contributed by fewer deletion events (Fig. 2c, Supplementary Fig. 13). These ancestry disparities remained significant even

185 after controlling for potential confounding features (Supplementary Fig. 13) as well as when

186 restricting the cohorts to smokers defined based on mutational signatures ${ }^{3}$ (Supplementary 187 Note, Supplementary Fig. 14). On the contrary, the copy number differences among non188 smokers were less significant even though the overall trend was maintained (e.g. ploidy, 189 Figure 2c). Overall, the results suggest that EAS LUADs had fewer genomic alterations and 190 were less complex in genomic profiles than EUR LUADs. 


\section{Mutational signatures in EAS LUADs}

192 Using the non-negative matrix factorization (NMF) algorithm ${ }^{49,50}$, we discovered "ageing"

193 (COSMIC Signature 1), "smoking" (Signature 4), and "APOBEC" (Signature 2) in the EAS

194 cohort (Supplementary Fig. 15). Using the relative contributions of these signatures, we

195 clustered patients into three signature groups (Fig. 2d). The "smoking" group significantly

196 correlated with male smokers, and more KRAS mutations, while the "ageing" group showed

197 the reverse pattern (Supplementary Fig. 16). Among smokers, EAS had higher proportion of

198 "ageing" signatures than EUR, while among non-smokers, the signature proportions were

199 similar across cohorts (Supplementary Fig. 17). To understand the mutational history of

200 tumorigenesis, we partitioned the mutations into early (clonal) and late (subclonal)

201 mutations $^{51}$ and compared the relative contributions of the three signatures (Methods). The

202 "APOBEC" signatures were enriched in late mutations ${ }^{52}$ while the "smoking" signature was

203 more prominent among early mutations in both cohorts (Supplementary Fig. 17). We further

204 analyzed the mutational strand asymmetries with regard to transcription-coupled repair

205 (transcription asymmetry) and DNA replication-associated mechanisms (replication

206 asymmetry) during mutagenesis ${ }^{53}$. Transcription asymmetry profiles were similar across the

207 two cohorts, with similar discrepancies seen between smokers and non-smokers

208 (Supplementary Fig. 18). However, replication asymmetry profiles were less concordant.

209 This result indicated different mutagenic processes related to DNA replication may be

210 involved in tumorigenesis across the two cohorts.

211 A novel inflammatory subtype in EAS LUADs

212 Previous studies on transcriptomic profiles of EUR LUADs using supervised clustering

213 methods identified three major transcriptomic clusters: Terminal Respiratory Unit (TRU),

214 Proximal Proliferative (PP) and Proximal Inflammatory (PI $)^{4,54,55}$. To identify robust

215 transcriptomic clusters across cohorts, we processed RNA-seq data uniformly for the 172

216 EAS and 249 EUR LUAD tumor samples and clustered them in each cohort using

217 (unsupervised) NMF method. In both EAS and EUR cohorts, the NMF algorithm suggested

218 two or three groups as the best clustering solutions (Supplementary Fig. 19). In the two-group

219 partition, a TRU and a non-TRU cluster were found in both cohorts (Fig. 3a, top), with

220 consistent expression patterns (Fig. 3b, Methods ${ }^{56}$ ). The TRU clusters showed better survival

221 (Fig. 3a) and were characterized by features closer to the normal tissue (Extended Data Fig.

222 2), including downregulations of proliferation-related pathways ${ }^{57}$ (Fig. 3c, Methods, 
223 Supplementary Fig. 20), lower TMB, and lower genome instability (Fig. 3c,e,g,

224 Supplementary Table 9, Supplementary Fig. 21-22), suggesting a conserved basal partition of

225 LUADs across cohorts.

226 To further understand the RNA clustering, we partitioned patients in each cohort into three 227 sub-clusters (Fig. 3a, bottom). In the EUR cohort, NMF successfully recovered the three sub228 clusters previously defined by supervised clustering ${ }^{4}$ (Supplementary Fig. 23). Comparing the 229 sub-clusters across cohorts, two of the EAS sub-clusters were highly similar to the EUR 230 "TRU" and "PI" sub-clusters, and thus were named accordingly ${ }^{4}$ (Fig. 3b, Supplementary Fig. 231 24). Notably, we found a novel RNA sub-cluster unique to the EAS cohort (Fig. 3b,f) with 232 up-regulation of inflammation-related genes ${ }^{58,59}$ and a higher level of immune infiltration ${ }^{57,60}$ 233 (Fig. 3d,g, Supplementary Table 10). Thus, we named this novel subtype as "TRU234 inflammatory" (TRU-I) since most of its members were among the TRU cluster in the two235 group partition (Fig. 3a,g).

236 Compared with other EAS sub-clusters, the TRU-I sub-cluster had the lowest level of TP53 237 mutation, purity and CNV (Fig. 3e, Supplementary Fig. 25). Decomposition of the immune 238 components from the transcriptomic data ${ }^{57}$ showed that the TRU-I sub-cluster had the highest 239 proportions of T-cells, macrophages, neutrophils, natural killer (NK) cells and monocytes 240 (Fig. 3d,e, Supplementary Fig. 25-26), though TRU-I did not differ significantly from TRU 241 regarding survival outcomes, TMB and number of drivers (Fig 3a,e, Supplementary Fig. 25).

242 In the EUR cohort, similar segregations on the inflammatory features were observed between

243 the PI sub-cluster and the EUR-specific PP sub-cluster, where the PP subgroup was more 244 immunologically suppressive (Fig. 3d,e, Supplementary Table 11, Supplementary Fig. 27-28, 245 Extended Data Fig. 3). These results suggested that inflammatory features formed an 246 important axis across cohorts that could stratify LUAD transcriptomes and might help 247 identify patients suitable for immunotherapy.

\section{Ancestry differences in therapeutic opportunities}

249 The profile of genomic alterations across the two cohorts shed light on the possible treatment 250 differences. One key pathway with many targetable genes including EGFR is the RTK/Ras 251 pathway. Integrating mutations and CNVs, significantly more somatic alterations in KRAS, $252 A L K$ and ERBB4 were found for smokers, as well as in MET for non-smokers when 253 comparing EUR with EAS (Fig 4a, Supplementary Fig. 29). This is concordant with the 254 higher genomic changes in EUR LUADs. On the contrary, EGFR mutations were 
consistently more prevalent in EAS regardless of the smoking status. Consequently, RTK/Ras

256 pathway was mutated more frequently in EUR for smokers, but this trend was reversed 257 among non-smokers (Fig 4b). Inspecting a set of hallmark oncogenic pathways ${ }^{61}$, the EUR 258 cohort showed higher alteration frequencies across all pathways for smokers (Fig 4b). 259 However, among non-smokers, significantly higher alteration rates were observed only for 260 TGF $\beta$ and NRF2 pathways in the EUR cohort (Fig 4b).

261 Since not all somatic changes can lead to targetable mutations, we compared these somatic 262 changes with OncoKB, a well-curated clinical and molecular database ${ }^{62}$. Across all targetable 263 somatic changes, EGFR alterations were the dominant genetic changes in both cohorts (Fig 264 4c). Even though EUR patients have a higher prevalence of amplifications in MET (2.4\% vs $265 \quad 0.33 \%)$ and $C D K 4(4.8 \%$ vs $2.3 \%)$ amplification, such alterations were relatively infrequent 266 and are still under ongoing drug development. In addition to targeted therapy, the advent of 267 immunotherapy has expanded the therapeutic repertoire for LUAD. Using GEP score (a pan 268 cancer predictor for response to immunotherapy ${ }^{10,11}$, Methods), we found significantly higher 269 GEP scores in EAS (Fig. 4d), suggesting a higher potential for immune checkpoint blockade 270 (ICB) treatment in this group of patients. Since patients having EGFR mutations tend to have 271 lower GEP scores (Fig. 4d) as well as poor response to ICB treatment ${ }^{63}$, we focused on the 272 EGFR wild type (WT) patients in both cohorts. Notably, the EAS TRU-I subtype consistently 273 showed higher $\mathrm{T}$ cell infiltration, PDL1 expression, and GEP score (Fig. 4e), suggesting the 274 TRU-I phenotype as a potential biomarker for selecting patients for ICB treatment.

275 Integrative analysis of multiple data layers

276 Predicting patient outcomes by integrating features from multiple layers remains a perennial 277 challenge in the field. In this study, several highly correlated clinical and molecular features 278 were found to stratify patient outcomes. For example, higher intra-tumor heterogeneity (ITH) 279 was thought to predict poor survival ${ }^{64}$, but in our EAS cohort, patients with EGFR mutations 280 tended to have higher ITH (measured as the percentage of late mutations ${ }^{51}$ or pLM, Methods) 281 and better survival outcomes (Supplementary Fig. 30). This highlighted the complex 282 interaction between driver status and tumor heterogeneity ${ }^{20}$. To methodically investigate the 283 correlations among multi-dimensional features, we curated a list of 24 features ranging from 284 the basic clinical and genomic status to features that could stratify patient outcome 285 (Supplementary Fig. 31-32, Methods). These features were then classified into four groups: 286 clinical, driver genes, molecular, and ITH-related (the last three were also termed "genomic" 
287 features). Plotting the correlation structure across these features, we discovered a complex correlation network in the EAS cohort (Fig. 5a, Supplementary Fig. 33, Methods). Notably, the TMB-related and CNV-related correlation clusters represented two major axes of genomic complexity which connect multiple data layers (Fig. 5a).

291 Previous pan-cancer studies have questioned the merit of large scale sequencing, reporting 292 that patient outcome could be better predicted by clinical features than by genomic features ${ }^{65}$. 293 Using univariate and multivariate Cox models, we evaluated the predictive power of multi294 layer features in our EAS cohort. Based on the univariate p-value and the importance in 295 multivariate models, clinical and driver genes were found to be the strongest predictors, 296 followed by molecular and ITH features (Fig. 5a,b, Supplementary Fig. 34, Methods). A 297 similar trend was seen when evaluating these feature groups individually using c-index for 298 their prediction accuracies (Fig. 5c, Methods). The ITH features generally performed the 299 worst, indicating that heterogeneity inferred from single sector data might be insufficient in 300 predicting patient survival ${ }^{66}$. Based on the predicted hazard from the multivariate Cox model, 301 we partitioned patients into three survival groups with different prognosis, and observed clear 302 segregation across multiple genomic features (Fig. 5e,f, Supplementary Fig. 35). While 303 clinical features were strong predictors, we found that genomic features also predicted patient 304

307 We observed many similar trends when we performed the same analysis on the EUR cohort: 308 a) EGFR mutant lung cancer also had higher ITH (Supplementary Fig. 30), b) a highly 309 correlated network structure among features with the TMB- and CNV-related clusters 310 (Supplementary Fig. 36, 37), c) Clinical features ranked top in predicting patient survival, 311 followed by driver genes (Fig. 5b,c, Supplementary Fig. 34, 38), d) genomic features 312 segregated clearly among different survival subgroups (Supplementary Fig. 39-41), and e) the 313 genomic features alone could stratify patients within early or late stage tumors (Extended 314 Data Fig. 4). The overall similarity suggested that the basic architectures of LUADs are quite 315 conserved across ancestry backgrounds. Despite these similarities, predictive accuracies of 316 survival outcome were generally higher in EAS than in EUR (Fig. 5c), even when controlling 317 for the differences in smoking status, sample size or mutation status of EGFR between the 318 two cohorts (Extended Data Fig. 5). Since EUR LUADs had more genomic changes, the poor 319 survival predictability in EUR might be a characteristic associated with unstable genomes. 
320 Comparing predictive accuracies between patients with different levels of genome stability, 321 we observed higher predictive accuracies for tumors with lower ploidy in both cohorts with 322 clinical or driver gene predictors (Fig. 5d). Thus, the more stable genomes in EAS LUADs 323 may account for their better predictive accuracies than EUR.

\section{Discussion}

325 With the large cohort size and uniform computational procedures, we systematically profiled 326 the genomic landscape of EAS LUADs and revealed striking differences from the EUR 327 LUADs (Extended Data Fig. 6). EAS LUADs were characterized by more stable genomes 328 with fewer genomic alterations. This difference is stronger among smokers than never 329 smokers. Comparing the transcriptomic profiles, EAS LUADs were found to harbor an 330 ancestry-specific inflammatory subtype with high potential for the ICB therapy. In the 331 integrative survival analysis, molecular features were found to offer important information 332 beyond clinical features in patient stratification. Moreover, EAS LUADs showed higher 333 prognostic prediction accuracy than EUR LUADs, possibly due to a less complex genomic 334 constitution.

335 Ancestry differences in cancer have long been recognized across several tissue types and 336 were explained by genetic predisposition ${ }^{67-69}$ and environmental factors such as lifestyle, and 337 viral/bacterial infections ${ }^{70-72}$. However, we found that smoking status was insufficient to 338 explain many ancestry differences in genomic profiles between the two cohorts 339 (Supplementary Fig. 3, Supplementary Fig. 13), matching earlier epidemiological studies ${ }^{73}$. 340 Thus, intrinsic genetic predispositions ${ }^{74,75}$ or other environmental factors ${ }^{73,76,77}$ could play 341 important roles in ancestry differences in lung cancer. Due to changes in lifestyle, the 342 incidence of LUAD among EUR females is also increasing, which was not fully explained by 343 smoking behavior ${ }^{78,79}$. Further studies to understand the factors determining ancestry 344 differences can provide unique insights to the biology of lung cancer.

345 Comparing transcriptomic subtypes across ancestry backgrounds, our analysis identified 346 unexpected similarities and differences. Both cohorts shared the basal separations between 347 TRU and non-TRU ${ }^{80}$, which also matched the first principle component of the transcriptomic 348 space (Fig. 3a). Such separations were characterized by the differential expression of 349 proliferation-related gene sets such as the G2M checkpoint, MYC targets, and mTORC1 350 signalling (Fig. 3g, Supplementary Table 9, Supplementary Fig. 20-22, Extended Data Fig. 3).

351 Further clustering showed separations along the inflammatory axis, resulting in two cohort- 
352 specific subtypes: the more immunologically active TRU-I sub-cluster in EAS, and the 353 immune-suppressed PP sub-cluster in EUR (Fig. 3f). Separation along this axis matched the 354 second principal component (Fig. 3a) characterized by the up-regulation of inflammation 355 gene sets, including inflammatory response and IFN $\gamma$ response (Fig. 3g, Supplementary Table 356 10-11, Supplementary Fig. 25, 27, 28, Extended Data Fig. 3). Based on these observations, 357 we propose the proliferation axis and the inflammation axis to segregate the LUAD 358 transcriptomic subgroups (Fig. 3f). The two cohort-specific sub-clusters (TRU-I and PP) 359 represent tumors located at the opposite quadrants of the transcriptomic landscape. The TRU360 I subgroup consists of the less proliferative but more inflammatory tumors while the PP 361 subgroup consists of more proliferative and less inflammatory tumors.

362 We further observed that some of the molecular features accounting for ancestry differences 363 were connected. It is known that tumors with high genome instability have high proliferation 364 profile (e.g. up-regulated cell cycle pathways) and low immune infiltration ${ }^{81,82}$. As EUR 365 LUADs are featured with higher genomic instability (i.e. higher TMB and CNV), the 366 ancestry differences in genome instability might have contributed to the two cohort-specific 367 transcriptomic subtypes: TRU-I (more stable genome, low proliferation and high immune 368 infiltration) and PP (unstable genome, high proliferation and low immune infiltration). 369 Moreover, the higher genome instability might also contribute to poor prognostic prediction 370 in the EUR cohort. Thus, the study of ancestry differences in lung cancer illustrates how 371 genomic changes with molecular and clinical phenotypes can be unified to generate a unique 372 resource, and forms the basis to uncover the factors leading to the ancestry differences 373 between EAS and EUR.

374 In terms of patient stratification and clinical utility, both the proliferation and inflammation 375 axes could offer important opportunities. The proliferation axis can stratify patient outcome 376 across cohorts even when considering potential confounding factors ${ }^{80}$. The inflammation 377 axis may also predict a patient's response to ICB therapy based on levels of immune 378 infiltration, expression of PDL1 and ICB response signatures (i.e. GEP). Delineating these 379 features may provide a rational for combination approaches targeting proliferating subtypes 380 (e.g. with chemotherapy or targeted therapies), or alternative immune cell types (e.g. 381 macrophages) and the tumor microenvironment (e.g. inflammatory cytokines) in the 382 inflammatory subtypes. For the EGFR mutant population, TRU-I was observed in $36.3 \%$ of 383 patients. With the consistently elevated levels of immune cells in the TRU-I group, future 384 combinatorial approaches addressing other immune cell types (e.g. macrophages, Fig. 4e) in 
385 addition to $\mathrm{T}$ cells are warranted. Moreover, although previous survival analyses have 386 purported clinical features to be more robust predictors of patient survival than genomic 387 phenotypes ${ }^{65}$, we found that genomic features can effectively supplement traditional clinical 388 features to stratify patient outcome and to tailor bespoke therapies based on individual risk 389 profiles. Approaches including deep learning based methods ${ }^{83}$ could further integrate this 390 multi-dimensional data to drive precision medicine programs in the future. 
392 This work was funded by Glaxo Wellcome Manufacturing Pte Ltd, the Agency for Science 393 Technology and Research (A*STAR) (GIS/15-IAF100), the National Medical Research 394 Council, Singapore (NMRC/OFLCG/002c/2018, NMRC/OFIRG/0064/2017, 395 NMRC/TCR/007-NCC/2013), the National Research Foundation, Singapore (NRF396 NRFF2015-04), the German Cancer Aid (70113510), and the Lung Cancer Consortium 397 Singapore (LCCS). LCCS is jointly supported by philanthropy (including Singapore 398 Millennium Foundation), institutional, and industrial grants. W. Z. is supported in part by the National Key R\&D program of China grant 2018YFC0910400 and 2018YFC1406902, and National Science Foundation of China (31970566). We thank Beijing Genomics Institute for providing the published sequencing data. We thank T. Zhang for contribution to genomic data analysis, C. T. J. Ong, Y. L. Lee, I. M. L. Chua and W. W. J. Soon for the next generation sequencing work, GIS Research Pipeline Development team for support on analysis pipelines, Yasuji Matsuoka for administrative support. We thank Y. Cun for helpful discussions.

Author Contributions

A.M.H., W.Z., and D.S.W.T. conceived the study, and B. Lim, W.L.T, E.-H.T. contributed to it. A.M.H. coordinated the genomics work. D.S.W.T. coordinated the clinical work. J.C. and W.Z. coordinated works on data analysis. J.C. performed the genomic data analysis, and H.Y., C.Q.T., B. Lu, J.J.S.A., J.Q.L., F.G.S., R.N., Y.Y.L., C.Z.J.P., K.P.C., Y.F.L. and J.L. contributed to it. A.W. contributed to analysis pipeline development. A.S.M.T. and L.B.A. performed nucleic acids extractions, exome library preparations, and fusion gene validation with contributions of F.G.S. who also performed SNV validation. A.T., with help of Z.W.A. and T.K.H.L., performed the sectoring and histology studies and led the pathological work. P.S.C. and P.Y.N. contributed to RNA-seq library preparations and sequencing. T.P.T.K., B.H.O., D.A., A.A.L.H., A.G., and C.W.T. performed the surgery and biopsy procedures while D.S.W.T., A.T., W.-T.L., C.K.T., L.W., and E.-H.T. coordinated patient tissue banking, specimen transfer, and clinical data curation. P.J.C., M.M.C., J.J.S.A. and A.J.S. implemented

419 the OncoSG data portal. L.S., Z.W.A. and J.P.S.Y. performed the multiplex immunohistochemistry. J.C., W.Z., A.M.H., D.S.W.T., C.L.C., and E.-H.T. interpreted the data and conceptualized the manuscript. J.C. created figures and F.G.S., C.Q.T., J.J.S.A., S.M., K.P.C., and W.Z. contributed to it. J.C. and W.Z. wrote the manuscript and D.S.W.T., 


\section{References}

425 1. Bray, F. et al. Global cancer statistics 2018: GLOBOCAN estimates of incidence and 426 mortality worldwide for 36 cancers in 185 countries. CA. Cancer J. Clin. 68, 394-424 (2018).

2. Cheng, T.-Y. D. et al. The International Epidemiology of Lung Cancer: Latest Trends, 429 Disparities, and Tumor Characteristics. J. Thorac. Oncol. 11, 1653-1671 (2016).

430 3. Imielinski, M. et al. Mapping the Hallmarks of Lung Adenocarcinoma with Massively $431 \quad$ Parallel Sequencing. Cell 150, 1107-1120 (2012).

432 4. The Cancer Genome Atlas Research Network. Comprehensive molecular profiling of 433 lung adenocarcinoma. Nature 511, 543-550 (2014).

434 5. Campbell, J. D. et al. Distinct patterns of somatic genome alterations in lung 435 adenocarcinomas and squamous cell carcinomas. Nat. Genet. 48, 607-616 (2016).

436 6. Kadara, H. et al. Whole-exome sequencing and immune profiling of early-stage lung 437 adenocarcinoma with fully annotated clinical follow-up. Ann. Oncol. 28, 75-82 (2017).

438 7. Garon, E. B. et al. Pembrolizumab for the Treatment of Non-Small-Cell Lung Cancer. $N$. $439 \quad$ Engl. J. Med. 372, 2018-2028 (2015).

440 8. Borghaei, H. et al. Nivolumab versus Docetaxel in Advanced Nonsquamous Non-Small441 Cell Lung Cancer. N. Engl. J. Med. 373, 1627-1639 (2015).

442 9. Fehrenbacher, L. et al. Updated Efficacy Analysis Including Secondary Population 443 Results for OAK: A Randomized Phase III Study of Atezolizumab versus Docetaxel in 444 Patients with Previously Treated Advanced Non-Small Cell Lung Cancer. J. Thorac. $445 \quad$ Oncol. 13, 1156-1170 (2018). 446 10. Ayers, M. et al. IFN- $\boldsymbol{\gamma}$-related mRNA profile predicts clinical response to PD-1 blockade. $447 \quad$ J. Clin. Invest. 127, 2930-2940 (2017). 
448 11. Cristescu, R. et al. Pan-tumor genomic biomarkers for PD-1 checkpoint blockade-based 449 immunotherapy. Science 362, eaar3593 (2018).

450 12. Tan, W.-L. et al. Novel therapeutic targets on the horizon for lung cancer. Lancet Oncol. $451 \quad$ 17, e347-e362 (2016).

452 13. Shigematsu, H. et al. Clinical and biological features associated with epidermal growth 453 factor receptor gene mutations in lung cancers. J. Natl. Cancer Inst. 97, 339-346 (2005).

454 14. Jain, A. et al. Impact of Smoking and Brain Metastasis on Outcomes of Advanced EGFR 455 Mutation Lung Adenocarcinoma Patients Treated with First Line Epidermal Growth 456 Factor Receptor Tyrosine Kinase Inhibitors. PloS One 10, e0123587 (2015).

457 15. Kris, M. G. et al. Using multiplexed assays of oncogenic drivers in lung cancers to select $458 \quad$ targeted drugs. JAMA 311, 1998-2006 (2014).

459 16. Clinical Lung Cancer Genome Project (CLCGP) \& Network Genomic Medicine (NGM).

460 A genomics-based classification of human lung tumors. Sci. Transl. Med. 5, 209ra153 $461 \quad$ (2013).

462 17. Wu, K. et al. Frequent alterations in cytoskeleton remodelling genes in primary and 463 metastatic lung adenocarcinomas. Nat. Commun. 6, 10131 (2015).

464 18. Wang, C. et al. Whole-genome sequencing reveals genomic signatures associated with 465 the inflammatory microenvironments in Chinese NSCLC patients. Nat. Commun. 9, 2054 $466 \quad$ (2018).

467 19. Luo, W. et al. Characteristics of genomic alterations of lung adenocarcinoma in young 468 never-smokers. Int. J. Cancer 143, 1696-1705 (2018).

469 20. Nahar, R. et al. Elucidating the genomic architecture of Asian EGFR -mutant lung 470 adenocarcinoma through multi-region exome sequencing. Nat. Commun. 9, 216 (2018).

471 21. McKenna, A. et al. The Genome Analysis Toolkit: a MapReduce framework for 472 analyzing next-generation DNA sequencing data. Genome Res. 20, 1297-1303 (2010). 
473 22. DePristo, M. A. et al. A framework for variation discovery and genotyping using next-

474 generation DNA sequencing data. Nat. Genet. 43, 491-498 (2011).

475 23. Ramos, A. H. et al. Oncotator: cancer variant annotation tool. Hum. Mutat. 36, E2423-

$476 \quad 2429(2015)$.

477 24. Dobin, A. et al. STAR: ultrafast universal RNA-seq aligner. Bioinformatics 29, 15-21

$478 \quad$ (2013).

479 25. Li, B. \& Dewey, C. N. RSEM: accurate transcript quantification from RNA-Seq data

$480 \quad$ with or without a reference genome. BMC Bioinformatics 12, 323 (2011).

26. Cibulskis, K. et al. Sensitive detection of somatic point mutations in impure and heterogeneous cancer samples. Nat. Biotechnol. 31, 213-219 (2013).

27. Saunders, C. T. et al. Strelka: accurate somatic small-variant calling from sequenced 484 tumor-normal sample pairs. Bioinformatics 28, 1811-1817 (2012).

28. Lawrence, M. S. et al. Mutational heterogeneity in cancer and the search for new cancerassociated genes. Nature 499, 214-218 (2013).

29. Tokheim, C. J., Papadopoulos, N., Kinzler, K. W., Vogelstein, B. \& Karchin, R. Evaluating the evaluation of cancer driver genes. Proc. Natl. Acad. Sci. U. S. A. 113, 14330-14335 (2016).

30. Nicorici, D. et al. FusionCatcher - a tool for finding somatic fusion genes in paired-end RNA-sequencing data. Preprint at bioRxiv https://doi.org/10.1101/011650 (2014).

31. Liu, L. et al. Comprehensive genomic profiling of lung cancer using a validated panel to 493 explore therapeutic targets in East Asian patients. Cancer Sci. 108, 2487-2494 (2017).

494 32. Hu, X. et al. TumorFusions: an integrative resource for cancer-associated transcript 495 fusions. Nucleic Acids Res. 46, D1144-D1149 (2018).

496 33. Weischenfeldt, J. et al. Integrative genomic analyses reveal an androgen-driven somatic 497 alteration landscape in early-onset prostate cancer. Cancer Cell 23, 159-170 (2013). 
34. Liao, S. et al. A genetic interaction analysis identifies cancer drivers that modify EGFR dependency. Genes Dev. 31, 184-196 (2017).

35. Gibson, B. A. \& Kraus, W. L. New insights into the molecular and cellular functions of poly(ADP-ribose) and PARPs. Nat. Rev. Mol. Cell Biol. 13, 411-424 (2012).

36. Ikeda, Y. et al. Germline PARP4 mutations in patients with primary thyroid and breast cancers. Endocr. Relat. Cancer 23, 171-179 (2016).

37. Long, N. P. et al. Novel Biomarker Candidates for Colorectal Cancer Metastasis: A Meta-analysis of In Vitro Studies. Cancer Inform. 15, 11-17 (2016).

38. Katsyv, I. et al. EPRS is a critical regulator of cell proliferation and estrogen signaling in ER+ breast cancer. Oncotarget 7, 69592-69605 (2016).

39. Wang, J.-W. et al. Deregulated expression of LRBA facilitates cancer cell growth. Oncogene 23, 4089-4097 (2004).

40. Sung, H. et al. Inactivation of RASA1 promotes melanoma tumorigenesis via R-Ras activation. Oncotarget 7, 23885-23896 (2016).

41. Zhu, Y.-J., Xu, B. \& Xia, W. Hsa-mir-182 downregulates RASA1 and suppresses lung squamous cell carcinoma cell proliferation. Clin. Lab. 60, 155-159 (2014).

42. Wang, W.-H., Studach, L. L. \& Andrisani, O. M. Proteins ZNF198 and SUZ12 are downregulated in hepatitis B virus (HBV) X protein-mediated hepatocyte transformation and in HBV replication. Hepatology 53, 1137-1147 (2011).

43. Tamborero, D. et al. Cancer Genome Interpreter annotates the biological and clinical relevance of tumor alterations. Genome Med. 10, 25 (2018).

44. Lee, Y. J. et al. Activating mutations within the EGFR kinase domain: a molecular predictor of disease-free survival in resected pulmonary adenocarcinoma. J. Cancer Res. Clin. Oncol. 135, 1647-1654 (2009). 
45. D’Angelo, S. P. et al. Distinct Clinical Course of EGFR-Mutant Resected Lung Cancers: Results of Testing of 1118 Surgical Specimens and Effects of Adjuvant Gefitinib and Erlotinib. J. Thorac. Oncol. 7, 1815-1822 (2012).

46. Jeon, J. H. et al. Prognostic and predictive role of epidermal growth factor receptor mutation in recurrent pulmonary adenocarcinoma after curative resection. Eur. J. CardioThorac. Surg. Off. J. Eur. Assoc. Cardio-Thorac. Surg. 47, 556-562 (2015).

47. Favero, F. et al. Sequenza: allele-specific copy number and mutation profiles from tumor sequencing data. Ann. Oncol. 26, 64-70 (2015).

48. Mermel, C. H. et al. GISTIC2.0 facilitates sensitive and confident localization of the targets of focal somatic copy-number alteration in human cancers. Genome Biol. 12, R41 (2011).

49. Gaujoux, R. \& Seoighe, C. A flexible R package for nonnegative matrix factorization. BMC Bioinformatics 11, 367 (2010).

50. Blokzijl, F., Janssen, R., van Boxtel, R. \& Cuppen, E. MutationalPatterns: comprehensive genome-wide analysis of mutational processes. Genome Med. 10, 33 (2018).

51. McGranahan, N. et al. Clonal status of actionable driver events and the timing of mutational processes in cancer evolution. Sci. Transl. Med. 7, 283 ra54 (2015).

52. Bruin, E. C. de et al. Spatial and temporal diversity in genomic instability processes defines lung cancer evolution. Science 346, 251-256 (2014).

53. Haradhvala, N. J. et al. Mutational Strand Asymmetries in Cancer Genomes Reveal Mechanisms of DNA Damage and Repair. Cell 164, 538-549 (2016).

54. Hayes, D. N. et al. Gene expression profiling reveals reproducible human lung adenocarcinoma subtypes in multiple independent patient cohorts. J. Clin. Oncol. Off. J. Am. Soc. Clin. Oncol. 24, 5079-5090 (2006). 
546 55. Wilkerson, M. D. et al. Differential Pathogenesis of Lung Adenocarcinoma Subtypes

547 Involving Sequence Mutations, Copy Number, Chromosomal Instability, and

$548 \quad$ Methylation. PLOS ONE 7, e36530 (2012).

549 56. Hoshida, Y., Brunet, J.-P., Tamayo, P., Golub, T. R. \& Mesirov, J. P. Subclass Mapping:

550 Identifying Common Subtypes in Independent Disease Data Sets. PLOS ONE 2, e1195

$551 \quad$ (2007).

552 57. Nirmal, A. J. et al. Immune Cell Gene Signatures for Profiling the Microenvironment of

$553 \quad$ Solid Tumors. Cancer Immunol. Res. 6, 1388-1400 (2018).

554 58. Subramanian, A. et al. Gene set enrichment analysis: A knowledge-based approach for

555 interpreting genome-wide expression profiles. Proc. Natl. Acad. Sci. 102, 15545-15550

$556 \quad(2005)$

557 59. Liberzon, A. et al. The Molecular Signatures Database (MSigDB) hallmark gene set

$558 \quad$ collection. Cell Syst. 1, 417-425 (2015).

559 60. Yoshihara, K. et al. Inferring tumour purity and stromal and immune cell admixture from $560 \quad$ expression data. Nat. Commun. 4, 2612 (2013).

561 61. Sanchez-Vega, F. et al. Oncogenic Signaling Pathways in The Cancer Genome Atlas.

$562 \quad$ Cell 173, 321-337.e10 (2018).

563 62. Chakravarty, D. et al. OncoKB: A Precision Oncology Knowledge Base. JCO Precis.

$564 \quad$ Oncol. 1-16 (2017) doi:10.1200/PO.17.00011.

565 63. Wu, Y.-L. et al. A consensus on immunotherapy from the 2017 Chinese Lung Cancer

566 Summit expert panel. Transl. Lung Cancer Res. 7, 428-436-436 (2018).

567 64. Morris, L. G. T. et al. Pan-cancer analysis of intratumor heterogeneity as a prognostic

568 determinant of survival. Oncotarget 7, 10051-10063 (2016).

569 65. Yuan, Y. et al. Assessing the clinical utility of cancer genomic and proteomic data across

$570 \quad$ tumor types. Nat. Biotechnol. 32, 644 (2014). 
571 66. Jamal-Hanjani, M. et al. Tracking the Evolution of Non-Small-Cell Lung Cancer. N. Engl.

$572 \quad$ J. Med. 376, 2109-2121 (2017).

573 67. Tan, D. S. W., Mok, T. S. K. \& Rebbeck, T. R. Cancer Genomics: Diversity and

574 Disparity Across Ethnicity and Geography. J. Clin. Oncol. 34, 91-101 (2016).

575 68. Haiman, C. A. et al. Genome-wide association study of prostate cancer in men of African

576 ancestry identifies a susceptibility locus at 17q21. Nat. Genet. 43, 570-573 (2011).

577 69. Haiman, C. A. et al. A common variant at the TERT-CLPTM1L locus is associated with

578 estrogen receptor-negative breast cancer. Nat. Genet. 43, 1210-1214 (2011).

579 70. Ziegler, R. G. et al. Migration Patterns and Breast Cancer Risk in Asian-American

$580 \quad$ Women. JNCI J. Natl. Cancer Inst. 85, 1819-1827 (1993).

581 71. Kuniholm, M. H. et al. Prevalence of hepatitis C virus infection in US Hispanic/Latino

582 adults: results from the NHANES 2007-2010 and HCHS/SOL studies. J. Infect. Dis. 209,

$583 \quad 1585-1590(2014)$.

584 72. Teng, A. M., Blakely, T., Baker, M. G. \& Sarfati, D. The contribution of Helicobacter

585 pylori to excess gastric cancer in Indigenous and Pacific men: a birth cohort estimate.

$586 \quad$ Gastric Cancer 20, 752-755 (2017).

587 73. Haiman, C. A. et al. Ethnic and Racial Differences in the Smoking-Related Risk of Lung

588 Cancer. N. Engl. J. Med. 354, 333-342 (2006).

589 74. Wu, C. et al. Genetic variants on chromosome 15q25 associated with lung cancer risk in

$590 \quad$ Chinese populations. Cancer Res. 69, 5065-5072 (2009).

591 75. Wang, J. et al. Genetic predisposition to lung cancer: comprehensive literature integration,

592 meta-analysis, and multiple evidence assessment of candidate-gene association studies.

593 Sci. Rep. 7, 8371 (2017). 
594 76. Seow, A. et al. Fumes from meat cooking and lung cancer risk in Chinese women.

595 Cancer Epidemiol. Biomark. Prev. Publ. Am. Assoc. Cancer Res. Cosponsored Am. Soc.

$596 \quad$ Prev. Oncol. 9, 1215-1221 (2000).

597 77. Lee, T. \& Gany, F. Cooking oil fumes and lung cancer: a review of the literature in the

598 context of the U.S. population. J. Immigr. Minor. Health 15, 646-652 (2013).

599 78. Lortet-Tieulent, J. et al. Convergence of decreasing male and increasing female incidence

600 rates in major tobacco-related cancers in Europe in 1988-2010. Eur. J. Cancer Oxf. Engl.

$601 \quad 1990$ 51, 1144-1163 (2015).

602 79. Jemal, A. et al. Higher Lung Cancer Incidence in Young Women Than Young Men in the

603 United States. N. Engl. J. Med. 378, 1999-2009 (2018).

604 80. Takeuchi, T. et al. Expression Profile-Defined Classification of Lung Adenocarcinoma

605 Shows Close Relationship With Underlying Major Genetic Changes and

606 Clinicopathologic Behaviors. J. Clin. Oncol. 24, 1679-1688 (2006).

607 81. Davoli, T., Uno, H., Wooten, E. C. \& Elledge, S. J. Tumor aneuploidy correlates with

608 markers of immune evasion and with reduced response to immunotherapy. Science $\mathbf{3 5 5}$,

$609 \quad$ eaaf8399 (2017).

610 82. Taylor, A. M. et al. Genomic and Functional Approaches to Understanding Cancer

611 Aneuploidy. Cancer Cell 33, 676-689.e3 (2018).

612 83. Coudray, N. et al. Classification and mutation prediction from non-small cell lung 613 cancer histopathology images using deep learning. Nat. Med. 24, 1559-1567 (2018). 


\section{$614 \quad$ Figure legends}

\section{Fig. 1. Driver genes for EAS LUADs}

616 (a) Cohort composition of the East Asian- (EAS) and European-ancestry (EUR) datasets in

617 this study. (b) Summary of the clinical and mutation burden differences across the two

618 cohorts. Asterisks are labelled according to FDR q-values calculated among (b). The

619 corresponding p-values testing differences in stage, gender, age and TMB for smokers and

620 non-smokers are: $\left(0.12,8.8 \times 10^{-13}, 0.088,0.0088\right)$ and $(0.029,0.75,0.046,0.041)$ respectively.

621 (c) Mutation profiles of the EAS cohort for driver genes detected by MutSigCV and 20/20+,

622 along with the fusion genes detected by FusionCatcher. The blue annotations on the right-

623 hand side of the gene names indicate whether the genes were called by MutSigCV and

624 20/20+. Red annotations denote which genes have been previously implicated as LUAD

625 drivers and whether it is in the cancer gene census (CGC) list. Mutant frequencies in the

626 cohort are shown on the left. Mutation burden (number of mutations per MB) for each patient

627 is shown on top. (d) Comparison of mutation frequencies of driver genes between EAS and

628 EUR cohorts among smokers and non-smokers, respectively. Only genes with significant (q-

629 value $<0.1$ ) ancestry differences calculated by two-sided Fisher's exact test are shown. (e)

630 Correlation between driver mutations and clinical phenotypes in the EAS cohort. Significant

631 correlations between driver mutation and clinical phenotypes are highlighted in red. Boxplot

632 definition of panel (b) and statistical tests used for other panels can be found under Online

633 Methods.

\section{Fig. 2. Copy number variation and mutation signature analysis}

635 (a) Chromosome arm-level copy number variation (CNV) frequencies in East Asian-ancestry

636 (EAS, n=302) and European-ancestry (EUR, n=249) cohorts. Dark red (amplifications) and

637 blue (deletions) represent the EAS cohort, while light colors represent the EUR cohort.

638 Significantly altered arms in each cohort are highlighted with black borders. Arms with

639 significant cohort differences were labelled with green dots. (b) Focal-level CNV across

640 chromosome 1-22 with y-axis presenting GISTIC FDR q-values. Selected genes in the cancer

641 gene census list are labelled in the significant peak regions shared by both cohorts (Methods).

642 Color schemes are the same as in 2a. (c) Comparison of the EAS (smoker $\mathrm{n}=111$; non-smoker

$643 \mathrm{n}=187$ ) and EUR (smoker $\mathrm{n}=172$; non-smoker $\mathrm{n}=68$ ) cohorts on copy number metrics: ploidy,

644 genome instability index (GII), GII calculated with only deleted regions (GII.Deletion), and

645 genome doubling (GD). Asterisks are labelled according to FDR q-values calculated among 
646 (c). Corresponding p-values testing differences in ploidy, GII, GII.Deletion, and GD for 647 smokers and non-smokers are: $(0.0023,0.0067,0.00028,0.0034)$ and $(0.59,0.82,0.16,1)$,

648 respectively. (d) Clustering of EAS patients based on their proportion of mutation signatures.

649 Each cluster group is named according to the dominant mutation signature (i.e., smoking,

650 ageing, and APOBEC). Boxplot definition of panel (c) and statistical tests used can be found 651 under Online Methods.

652 Fig. 3. Transcriptomic clusters in East Asian- and European-ancestry cohorts.

653 (a) Principal component analysis (PCA) and survival analysis of the transcriptomic clusters 654 found in the East Asian-ancestry (EAS) (left) and European-ancestry (EUR) (right) cohorts 655 when partitioning the cohorts into two clusters (top) or three sub-clusters (bottom). (b)

656 Subclass mapping of the subtypes found in EAS and EUR (Methods). Significant

657 correspondences between clusters are highlighted in red with Bonferroni-adjusted p-values

658 (Methods). Sample sizes are the same as in the PCA plot in (a). (c) Phenotypic differences

659 between the two clusters (TRU and non-TRU); and (d) phenotypic differences among the

660 three sub-clusters focusing on immune-related features. Asterisks are labelled according to

661 FDR q-values calculated among (c) and (d) together. (e) Summary of the phenotypic

662 differences between the two clusters (TRU \& non-TRU), and the two sub-clusters along the

663 inflammation axis (TRU-I \& TRU in EAS; PP \& PI in EUR) (Methods). Features with

664 significant differences are highlighted in red (indicating higher values) and blue (indicating

665 lower values), while non-significant (n.s.) features are coloured grey. Sample sizes are the

666 same as in (b) except for TMB, Num.Driver, GII, GD, TP53, EGFR, and Purity in the EAS

667 two-cluster comparisons (non-TRU, n=82; TRU, n=87). (f) Subtype correspondence across

668 cohorts. The TRU-I sub-cluster is EAS-specific, and PP sub-cluster is EUR-specific. The

669 putative expression levels of proliferation- and inflammation-related genes are annotated at

670 the side. (g) Phenotypes of the RNA sub-clusters in EAS. The top two rows indicate the

671 cluster assignment of the patients. The following rows show the normalized mean expression

672 of GSEA enriched gene sets from the differential expressed genes between the TRU and non-

673 TRU clusters and between the TRU-I and TRU sub-clusters, and the values of immune-

674 related features. High values were shown in red and low in blue. Boxplot definition and

675 statistical tests used can be found under Online Methods.

676 Fig. 4. Ancestry differences in therapeutic opportunities. 
677 (a) Comparison of the alteration frequency (including mutation, fusion and CNV) of genes in 678 the RTK/RAS pathway across the two cohorts for smoker (East Asian ancestry (EAS), $\mathrm{n}=111$; 679 European ancestry (EUR), n=172) and non-smokers (EAS, n=187; EUR, n=68). Genes with 680 significant cohort differences were labelled with asterisk (two-sided Fisher's exact test). (b) 681 Comparison of the alteration frequency of genes in ten hallmark pathways across the two 682 cohorts. Differences with significant p-values were labelled (two-sided Fisher's exact test). 683 Sample sizes are the same as in (a). (c) Comparison of the druggable mutations frequency 684 across the two cohorts. The OncoKB evidence levels (Lv) annotated after gene names 685 indicate the evidence level of the druggable target. Lv1, FDA-recognized biomarker in 686 LUAD; Lv2A, standard care biomarker for drug in LUAD; Lv2B, standard care biomarker 687 for drug in another indication. (d) Comparison of the GEP score across different cohorts. 688 Horizontal dotted line denotes the cut-off value -0.318 from pan cancer study, which 689 segregates patients that are responsive to ICB (higher) or non-responsive (lower). (e) T cell 690 infiltration, GEP score, PDL1 expression in wildtype EGFR samples across the three EAS 691 RNA subtypes. (f) Expression levels of possible alternative ICB targets across the three EAS 692 RNA subtypes. Asterisks are labelled according to FDR q-values calculated among (e) and (f). 693 Boxplot definition and statistical tests used can be found under Online Methods.

\section{Fig. 5. Survival groups and cohort differences.}

695 (a) Correlation and prognosis importance for 24 features in the East Asian-ancestry (EAS) 696 cohort ( $\mathrm{n}=271$, patients with no missing data). Node sizes and node borders denote the hazard 697 ratios and significance in the univariate Cox models, respectively. Lines connect significantly 698 correlated features (FDR q-value $<0.01$ ) with thickness representing FDR q-values. (b) Top:

699 feature importance in multivariate Cox models (percentages of the Wald statistic; EAS, $700 \mathrm{n}=271$; EUR, $\mathrm{n}=218$ ). Bottom: total importance for each feature group. (c-d) Comparisons of 701 prediction accuracies of multivariate Cox models using various features. Accuracies were 702 calculated as Harrell's concordance index (c-index) with $\mathrm{n}=100$ random splitting of training 703 and test sets. (c) p-values for all, clinical, driver, molecular, and ITH features are $1.9 \times 10^{-15}$,

$7041.6 \times 10^{-7}, 0.021,0.017$, and 0.034 , respectively. (d) Comparisons between high and low 705 genome instability levels. p-values in EAS and European-ancestry (EUR) cohort for all, 706 clinical, and driver features are $(1.6 \times 10-15,0.0058),\left(<2.22 \times 10^{-16},<2.22 \times 10^{-16}\right)$, and $707\left(<2.22 \times 10^{-16}, 0.14\right)$, respectively. Asterisks denote FDR q-values calculated among (c) and 708 (d). (e) Clinical and genomic phenotypes of the survival groups in the EAS cohort. (f) 709 Kaplan-Meier curves of the survival groups in the EAS cohort for all patients, patients of 
710 stage I \& II, and patients of stage III \& IV, respectively. For (e-f), group sizes are 91, 90, and

71190 for S1, S2, and S3, respectively. Boxplot definition and detailed statistical tests used in all

712 panels can be found under Online Methods.

\section{Online Methods}

715 Patient recruiting, sample sequencing and public data processing

716 Patients with lung adenocarcinoma were recruited from the National Cancer Center of

717 Singapore (NCCS) with written informed consent. All tumor specimens were reviewed by

718 pathologists to determine the histological subtype, histological grade as well as TNM staging

719 (Supplementary Table 1). Tumor and adjacent normal tissues were harvested by pathologists

720 following surgical resection and biopsy, respectively. Adjacent normal lung tissue or blood 721 was used as a matched normal control. DNA and RNA were extracted using AllPrep 722 DNA/RNA/miRNA Universal kit (Qiagen). For WES, 200 to $1000 \mathrm{ng}$ of genomic DNA was 723 sheared to shorter fragments using the Covaris system, and then subjected to library 724 preparation using NEBnext End repair, A-tailing and Ligation modules (New England 725 Biolabs), with a partially double-stranded custom adaptor (Supplementary Table 12), 726 followed by PCR with i5 and i7 index primers to generate dual-indexed libraries. Six dual727 indexed libraries were pooled in each capture with the SeqCap EZ Human Exome v3.0 kit 728 (Nimblegen). Captured DNA was washed, purified, and amplified, then subjected to $2 \times 151$ 729 bp sequencing in 1 lane on the HiSeq 4000 sequencer (Illumina). For RNA-seq, 1.2 to $4 \mu \mathrm{g}$ of 730 total RNA was used for library preparation using the TruSeq Stranded mRNA Sample 731 Preparation Kit (Illumina), with fragmentation at $80^{\circ} \mathrm{C}$ for 2 minutes. 24 samples were pooled 732 into a single multiplex library, and each library was subjected to 2 x 101 bp sequencing in 733 three lanes on the HiSeq 2000 sequencer (Illumina) with V4 reagents.

734 Raw RNA-Seq fastq and WES BAM files of 272 patients from TCGA LUAD project were 735 downloaded from TCGA legacy archive (including 229 patients from the first stage of TCGA 736 LUAD $^{4}$ and all the 43 accessible never smoker patients from TCGA LUAD cohort), and raw 737 WES and WGS BAM files of 92 patients from the BGI cohort were downloaded from EGA 738 (Supplementary Note, Supplementary Table 1). The ancestry of each patient was inferred 739 using data from Human Genome Diversity Project ${ }^{84}$, and 22 patients of non-European origin 740 were excluded from the TCGA cohort (Supplementary Note, Supplementary Fig. 2). One 
741 patient from the TCGA cohort was further excluded due to the exceptionally high number of somatic variants compared to the published reports (Supplementary Note).

\section{Somatic variant identification}

744 For the Singapore and BGI cohort, short sequence reads were mapped to the human reference 745 genome GRCh37 (GATK Resource Bundle version 2.8) using BWA-MEM (version 0.7.12) ${ }^{85}$ 746 with default parameters. Following GATK best practice ${ }^{21,22}$, PCR duplicates were first 747 removed and subsequently realigned and recalibrated (available at https:/github.com/gis$748 \mathrm{rpd} /$ pipelines, GATK version 3.1.1). Similar steps including marking duplication, realignment 749 and recalibration were performed for the BAM files from the TCGA cohort. Somatic single750 nucleotide variants (SNVs) were identified using $\mathrm{MuTect}^{26}$ (version 1.1.7). To reduce false 751 positive calls, a panel of normal was created for each cohort of the same library preparation 752 kit, using all normal samples in the same cohort. Tumor samples were then used to call 753 somatic mutations against the paired normal and filtered with the corresponding panel of 754 normal. In addition, based on the validation results (see below), we further filtered out 755 mutations that tend to be false positives, retaining only the ones supported by at least 3 756 mutation reads with a variant allele frequency (VAF) larger than 0.08. Somatic insertions and 757 deletions (indels) were called using Strelka $^{27}$ (version 1.0.15) with default parameters.

758 Variant validation by targeted capture deep sequencing.

759 To validate variants identified by whole exome sequencing (WES), we randomly selected 55 760 variants from 7 patients (a total number of 385 variants of which 370 were SNVs and 15 were 761 indels) and designed a pool of customized PCR primers for targeted amplification and deep 762 sequencing. Libraries were constructed based on PCR amplicons and sequenced using 763 Illumina MiSeq platform to a mean coverage of 5,941 at targeted sites. The sequencing reads 764 from the validation data flowed through a similar procedure as the WES sequencing. A 765 somatic variant was considered as validated if it met the following criteria: (1) the same 766 alternative allele at the same position was observed in both WES and deep-sequencing at the 767 target position (2) the variant allele frequency (VAF) in the tumor was $>0.02$; (3) the VAF in 768 the normal was $<0.01$. Of the 385 targeted variants, 18 of them failed either in amplification 769 or sequencing. In the remaining 367 variants, 3 of them were found to be germline variants 770 with the detectable frequencies (VAF is $>0.02)$ in both tumor and normal DNA. Another 17 771 were found to be false positive with $\mathrm{VAF}<0.01$ in both tumor and normal DNA. We thus 772 obtained a raw validation rate of $94.6 \%$ (347 out of 367 ). To reduce potential false positive 
773 variants, we updated our variant calling pipeline with additional filters (Supplementary Note).

774 After this adjustment, 338 out of 348 somatic variants were successfully validated, with an 775 adjusted validation rate of $97.13 \%$.

\section{Driver gene identification and driver frequency comparison}

777 All somatic variants were first annotated using Oncotator ${ }^{23}$ (version 1.9.2). In order to 778 identify driver mutations for the EAS cohort, MutSigCV ${ }^{28}$ (version 1.41), as well as 20/20+ ${ }^{29}$ 779 (version 1.1.2), were used to infer the significantly mutated driver genes $(\mathrm{q}<0.1$ in any caller 780 and number of non-silent mutation $>=5$ ). We annotated LUAD drivers with a combination of 781 driver lists: a) significantly mutated genes in LUAD from nine publications $\mathrm{s}^{3,4,5,17,28,86-89}$; b) 782 drivers list from a PanCancer and PanSoftware analysis ${ }^{90}$, subsetting to only genes implicated 783 as lung adenocarcinoma related; c) the novel drivers identified from the EAS cohort in this 784 study. To compare the driver frequencies between different cohorts, the numbers of patients 785 containing non-silent mutations for each gene were curated in each cohort (Fig. 1b), with one 786 patient excluded who harboured 30 mutations of APC. To identify drivers with significant 787 cohort differences, we included only drivers with high mutation frequency in each cohort 788 (smokers: $>9$ patients in either cohort and with cohort difference of at least 1-fold change; 789 non-smokers: $>7$ in EAS or $>4$ in EUR). Two-sided Fisher's exact tests were then performed 790 and a false discovery rate (FDR) cutoff of 0.1 was used for reported genes.

791 Fusion gene discovery and validation

792 Fusion genes and their respective fusion points were predicted by FusionCatcher ${ }^{30}$ (version 793 0.99.5a beta) with the RNA-seq data. Fusion genes called from the tumor samples were 794 compared against those from normal samples to remove germline fusion alterations. In 795 addition, genes annotated as "probably false-positive" by FusionCatcher were also excluded. 796 The resulting fusion genes were further filtered and subsequently subject to experimental 797 validation by RT-PCR (Supplementary Note). Only validated fusions were used for 798 downstream analysis. For Fig. 4, fusion genes of the EUR cohort were identified from the 799 TumorFusions database ${ }^{32}$.

\section{$800 \quad$ Identification of copy number alterations}

801 For both EAS and EUR cohorts, Sequenza ${ }^{47}$ (version 2.1.2) was used to estimate the copy 802 number profile (including allele-specific copy number) as well as the tumor purity, ploidy for 803 each patient. Default settings were used following the recommendations of the manual. 
804 Genome doubling (GD) status for each sample was inferred by EstimateClonality ${ }^{51}$ (version

805 1.0) based on the copy number profile inferred by Sequenza. The genome instability index 806 (GII) was calculated as the percentage of a tumor genome showing different copy number 807 from the per-base median of that genome (all copy number values are integers as inferred by

808 Sequenza). GISTIC ${ }^{48}$ (Genome Identification of Significant Targets in Cancer, version 2.0.23) 809 was used to identify significantly amplified and deleted regions in each cohort. Output 810 segmentations from Sequenza were used as the input for GISTIC. Specifically, the Seg.CN 811 required by GISTIC was calculated with the depth.ratio estimation from Sequenza as 812 Seg. $\mathrm{CN}=\log _{2}(2 \times$ depth.ratio $)-1$. To avoid the bias from different sample ploidy, depth.ratios 813 were further divided by the ploidy of each sample to correct for ploidy. The GISTIC 814 parameters were set as follows: -genegistic 1 -smallmem 1 -broad 1 -brlen 0.5 -conf 0.95 815 armpeel 1 -savegene 1 -gcm extreme. Chromosome arms were labelled as "altered" in each 816 cohort if their GISTIC q-value $<0.1$. To identify arm-level CNV differences between cohorts, 817 Fisher's exact test were used followed by FDR correction for amplifications and deletions, 818 respectively, and significant difference was determined when an arm was: a) "altered" in both 819 cohorts and Fisher's exact test FDR < 0.1; or b) "altered" in only one cohort (Fig. 2a). Peak 820 regions with FDR q-value $<0.25$ were called as significant peaks and were annotated with 821 the Cancer Gene Census (version 85) (Supplementary Fig. 11, 12). To annotate the common 822 peaks in both EAS and EUR cohorts, peak regions with more than $50 \%$ overlap across 823 cohorts were merged and only genes within the overlapping regions were annotated (Fig. 2b).

\section{Mutational signatures identification and clustering}

825 In order to uncover mutational processes active in the LUAD patients, the $\mathrm{NMF}^{49}$ R package 826 (version 0.21.0) was used for de novo discovery of mutation signatures associated with the 827 EAS cohort. The MutationalPatterns ${ }^{50}$ (version 1.6.2) R package was used to characterize and 828 visualize the results from the de novo signature inference from NMF. The resulting signature 829 contribution proportions were used to cluster samples using the hierarchical clustering with 1830 cosine similarity distance and average linkage function. Fisher's Exact Tests were used to 831 find significant clinical co-occurrences with the signature groups. The same process was 832 performed on the TCGA cohort.

833 To survey the histories of the different mutational processes, we performed clonality analysis 834 on mutational signatures. Mutations in each patient were separated into early or late 835 mutations based on their copy number and cancer cell fraction (see Clonality Analysis below). 
836 Mutations in each category were then assigned to different signatures as previously identified

837 by NMF in each sample to estimate proportions of each signature. To confirm the findings

838 from these signature assignments, we also employed de novo decomposition ${ }^{91,92}$ for early and

839 late mutations separately without using the previously identified signatures, and the results

840 were concordant (Supplementary Fig. 17). To explore the mutational strand asymmetry

841 profiles, MutationalPatterns was used to dissect both the transcriptional and replicative strand

842 bias.

843 RNA clustering using non-negative matrix factorization (NMF)

844 Raw RNA sequence reads from the 172 tumors and 88 normal samples from 181 patients of 845 the EAS cohort and 220 tumor-normal paired samples of the EUR cohort were aligned to the 846 reference genome (UCSC hg19 with annotations from GENCODE v19) using STAR ${ }^{24}$ 847 (version 2.5.2a). Gene expression was subsequently quantified using RSEM ${ }^{25}$ (version 1.3.0).

848 The estimated expression counts from RSEM were then normalized using DESeq $2^{93}$ (version 849 1.16.1) followed by log transformation (adding 1 pseudo count and $\log 2$ transformation). For 850 clustering of the tumor samples, the top 3,000 most variable coding genes (based on median 851 absolute deviation) were chosen from the expressed genes (genes with at least 10 samples 852 showing non-zero counts) among tumor samples in each cohort. In order to identify robust 853 clusters, an unsupervised clustering method known as non-negative matrix factorization 854 (NMF), was used (NMF R-package version $0.21 .0^{49}$ ). Optimal rank parameters were first 855 determined using 50 runs of random starting points with default settings, followed by 300 856 runs with the optimal ranks to get the final NMF clustering solutions. Silhouette analysis was 857 performed to assess the consistency of the clustering solutions. The clustering solution of 858 rank=4 in the EAS cohort showed a low average silhouette value $(<0.6)$ and was excluded 859 from further analysis (Supplementary Fig. 19).

\section{Principal component and survival analysis of the RNA subtypes}

861 Based on the same set of 3,000 genes used in the NMF clustering, principal component 862 analysis (PCA) was performed using R built-in function prcomp. PCA plots were generated 863 using the first two PCs and sample points were coloured based on their NMF clusters. 864 Survival outcomes of each NMF cluster were estimated by the Kaplan-Meier method and the 865 log-rank test was used to calculate the p-values.

\section{Subclass mapping of the RNA subtypes}


867 To compare the clusters and sub-clusters identified from both EAS and EUR cohorts, an 868 unsupervised subclass mapping method SubMap $^{56}$ (version 3) was used to identify correspondence or commonality of subtypes from the two cohorts. SubMap analysis was performed on the GenePattern online platform using default settings with a random seed of 12345. The genes used were the intersection of the genes selected for the NMF clustering

$872(3,000$ most variable genes in each cohort) from the two cohorts. Significant correspondences 873 were identified with a cut-off of Bonferroni adjusted p-value $<0.01$ (Fig 3b, blocks with p874 value annotations).

\section{GSEA and immune profile analysis of the RNA subtypes}

876 DESeq2 with default settings was used for identifying differentially expressed genes and 877 determining their log fold change between NMF clusters. For gene set enrichment analysis 878 (GSEA $)^{58}$, a pre-ranked list of genes was generated based on the log fold change. Using fgsea 879 R package (version 1.2.1) ${ }^{94}$, GSEA was performed with the hallmark gene set (version 6.2) 880 from MSigDB ${ }^{59,95}$. The significantly enriched gene sets were filtered based on a cut-off of q881 value $<0.01$.

882 To decompose the immune components of tumor samples, the imsig R package (version 1.0.0) 883 was employed which uses a set of immune gene signatures generated by a network-based 884 deconvolution approach ${ }^{57}$. The imsig also provides gene signatures of biological processes 885 such as proliferation and interferon response, named "proliferation.module" and 886 "interferon.module" (Fig 3e). To further evaluate if the RNA subtypes would have different 887 responses to immune therapy, we compared the expression levels of PD1 (PDCD1), PDL1 888 and CTLA4, the immune infiltration levels and the immune evasion levels. The ESTIMATE R package $^{60}$ (version 1.0.13) was used with default settings to decompose the immune component from the expression counts, resulting in the immune score where higher score

891 denotes higher immune component in a tumor. To predict the responses to ICB therapy for 892 patients in our study, an 18-gene T cell-inflamed gene expression profile (GEP) were used, 893 and a GEP score for each sample was calculated as a weighted sum of these 18 genes after 894 normalization with 11 housekeeping genes following previously published methods ${ }^{10,11}$. To 895 confirm these findings with the expression level of proteins, we further performed multiplex 896 immunohistochemistry/immunofluorescence for CD8, CD68, CD3, and PDL1 in a subset of 89737 tumor samples (Supplementary Note).

\section{Clonality Analysis}


899 To determine the clonality of each mutation, i.e. if the mutation occurs early or late in tumor 900 evolution, EstimateClonality ${ }^{51}$ (version 1.0) was used to assign each somatic mutation as 901 either early or late based on its mutation copy number and cancer cell fraction. Only 902 mutations with a minimum VAF of 0.05 , minimum variant read depth of 5 , and minimum 903 total read depth of 30 were used. The proportion of late mutations (pLM) were calculated for 904 each patient by calculating the proportion of late mutations over total mutations (early plus 905 late), which can be used as an estimation of intra-tumor heterogeneity (ITH).

906 In addition to the pLM, we adopted three other metrics: number of clones (Num.Clone), 907 Shannon Index (SI), and MATH score. The number of clones was calculated by EXPANDS ${ }^{96}$ 908 (version 2.0.0) using the variants called by Mutect and the depth ratio of each mutation 909 inferred by Sequenza with the parameter $\max S$ set to 2.5. Based on the clone proportion

910 estimated by EXPANDS, we further calculated the Shannon Index ${ }^{97}$ using the formula

$911 S I=-\sum_{i=1}^{\text {Num.Clone }} p_{i} \ln p_{i}$, where $p_{i}$ is the proportion of clone $i$. MATH score ${ }^{98}$ is calculated

912 using the formula $M A T H_{i}=\frac{M A D\left(V A F_{i}\right)}{\operatorname{Median}\left(V A F_{i}\right)} * 100$, where $\mathrm{VAF}_{\mathrm{i}}$ is a vector of the VAF of all

913 mutations from sample $i$, and MAD stands for median absolute deviation. See supplementary

914 Note for detailed descriptions of these ITH metrics.

\section{Correlation of multilayer survival features}

916 A list of 24 features was curated which includes features of high importance in describing the 917 clinical or genomic profiles, and those found to have stratifying effects on patient outcome. 918 Stage, gender, age and smoker were included as basic clinical features. Driver genes that 919 show significant stratifying ability in either EAS or EUR cohort were also included. All the 920 four ITH features (pLM, number of clones, Shannon index and MATH score) were included 921 to represent different aspects of ITH. Molecular features are macro-level summary statistics 922 which provide overall descriptions of the genomic features, such as total tumor mutation 923 burden (TMB), genome doubling (GD), genomic instability index (GII), genome doubling 924 (GD) status, tumor ploidy and purity. Features that can stratify patient outcome were also 925 included such as number of drivers (Supplementary Fig. 31, 38) and signature groups. The 926 effect of TKI treatment was examined but it was not included in the survival analysis, 927 because only $36.6 \%$ of the EGFR mutant carriers received TKI and it did not show significant 928 effect in the models in the EAS cohort (Supplementary Fig. 9, 32). 
929 The pairwise correlations of all features were surveyed using various statistical tests and the

930 FDR q-values were depicted as solid lines with various widths on Fig. 5a or reported on plots

931 (Supplementary Fig. 33, 36, 37). For pairs of continuous variables, Student's t-test of the

932 linear regression coefficient was used for testing the significance. For pairs of categorical

933 variables, Fisher's exact test or Chi-squared test was used. For mixed pairs of categorical and

934 continuous variables, Kruskal-Wallis test was used. FDR q-values were calculated from all

935 correlation p-values within a cohort. Only correlations with FDR q-value $<0.01$ are plotted

936 on Fig. 5a. The weighted degree of connectivity of each group is calculated by averaging the

$937-\ln (F D R)$ for all significant pairs within a group.

\section{Feature importance and prediction accuracy in survival analysis}

939 Several important statistics weighting feature importance in predicting patient survival were

940 calculated. The simplest ones are the hazard ratio and the p-value of a feature in a univariate

941 Cox model (Fig. 5a). To evaluate the importance in multivariate models, Cox proportional

942 hazard models were used to represent linear and non-linear models. The Cox models were

943 fitted using the $c p f$ function in the rms R package with default parameters. The importance of

944 each feature for the cox model was determined by the proportion of the Wald statistic of each

945 feature over the sum of all Wald statistics of the model. As an approximation of the

946 "adequacy index", this proportion of the Wald statistic indicates the proportion of

947 explainable log-likelihood contributed by a feature.

948 To examine the prediction accuracy of each type of features, the Harrell's concordance 949 index $^{100}$ (c-index, calculated by survcomp R package ${ }^{101}$ ) were used (Fig 5). To gain the

950 estimate distributions of c-index, samples were randomly separated into training $(80 \%)$ and 951 test $(20 \%)$ sets for 100 times, a process similar to a previous study ${ }^{65}$. For fair comparisons

952 across the two cohorts, the proportion of smokers were balanced by randomly down-sampling 953 non-smokers in the EAS cohort to be the same as the number of non-smoker in the EAS 954 cohort, and down-sampling smokers in the EUR cohort similarly (Extended Data Fig. 5). To 955 better understand the differences among patients with good and bad outcomes, patients within 956 the same ancestry cohort were divided evenly into three survival groups based on the 957 predicted hazard from the multivariate Cox model with 24 features.

\section{Statistics and Reproducibility}

959 Unless specified otherwise, two-sided Fisher's exact test were used for the p-value 960 calculations between two categorical variables, while two-sided Wilcoxon rank sum test were 
961 used between two continuous variables for all figures. Log-rank tests were used for 962 comparing survival distributions in Kaplan-Meier plots. Two-sided t-tests were used for p963 values of the coefficients in all linear, logistic and Cox regression models. For multiple 964 testing correction, FDR corrections were performed unless specified otherwise. For all 965 boxplots or boxplots within violin plots, the box indicates the interquartile range (IQR); 966 middle line indicates median; whiskers indicate the highest and lowest values within $1.5 \times \mathrm{IQR}$ 967 away from the box; and dots plots values more than $1.5 \times \mathrm{IQR}$ away from the box. For 968 boxplots with notch, the notches extend to $\pm 1.58 \times \mathrm{IQR} / \operatorname{sqrt}(\mathrm{n})$ of the median where $\mathrm{n}$ is 969 sample size. For Fig. 1b panel TMB and for cohort comparison of number of drivers in the 970 first paragraph under section "Driver genes in EAS LUADs", the p-values were calculated 971 with one-sided t-test. For Fig. 1e the correlation between driver genes and clinical 972 phenotypes, we separate patients into younger ( $<=64 \mathrm{yrs}$, median age in the EAS cohort) and 973 older ( $>64$ yrs) groups, and then employed the one-sided Fisher's exact test to determine if 974 the mutation of a driver was enriched in a certain group using a $2 \times 2$ contingency table. Then, 975 FDR p-value correction was performed and correlations were reported when FDR $<0.1$. The 976 q-values were also reported in the main text. See the Life Sciences Reporting Summary for 977 further information on the reproducibility. 
979 Raw sequencing data have been deposited in the European Genome-phenome Archive (EGA, 980 http://www.ebi.ac.uk/ega/) under accession codes EGAD00001004421 and 981 EGAD00001004422. All clinical records, somatic mutations, copy number variations and 982 histological images from our study are hosted in OncoSG (https://src.gisapps.org/OncoSG/) 983 under data set "Lung Adenocarcinoma (GIS, 2019)" which is publicly available 984 (Supplementary Note).

985

986 Competing interests

987 A.M.H, D.S.W.T., and W.Z. received research funding by Glaxo Wellcome Manufacturing 988 Pte Ltd.

989 
990 References (Methods)

991 84. Cann, H. M. et al. A Human Genome Diversity Cell Line Panel. Science 296, 261-262

$992 \quad$ (2002).

993 85. Li, H. Aligning sequence reads, clone sequences and assembly contigs with BWA-MEM.

994 Preprint at arXiv https://arxiv.org/abs/1303.3997v2 (2013).

995 86. Ding, L. et al. Somatic mutations affect key pathways in lung adenocarcinoma. Nature

$996 \quad 455,1069-1075(2008)$.

997 87. Govindan, R. et al. Genomic Landscape of Non-Small Cell Lung Cancer in Smokers and

$998 \quad$ Never-Smokers. Cell 150, 1121-1134 (2012).

999 88. Lawrence, M. S. et al. Discovery and saturation analysis of cancer genes across 21

$1000 \quad$ tumour types. Nature 505, 495-501 (2014).

1001 89. Kandoth, C. et al. Mutational landscape and significance across 12 major cancer types.

$1002 \quad$ Nature 502, 333-339 (2013).

1003 90. Bailey, M. H. et al. Comprehensive Characterization of Cancer Driver Genes and

$1004 \quad$ Mutations. Cell 173, 371-385.e18 (2018).

1005 91. Tan, V. Y. F. \& Févotte, C. Automatic Relevance Determination in Nonnegative Matrix

1006 Factorization with the /spl beta/-Divergence. IEEE Trans. Pattern Anal. Mach. Intell. 35,

$1007 \quad 1592-1605(2013)$.

1008 92. Kim, J. et al. Somatic ERCC2 mutations are associated with a distinct genomic signature

1009 in urothelial tumors. Nat. Genet. 48, 600-606 (2016).

1010 93. Love, M. I., Huber, W. \& Anders, S. Moderated estimation of fold change and dispersion

1011 for RNA-seq data with DESeq2. Genome Biol. 15, (2014).

1012 94. Korotkevich, G., Sukhov, V. \& Sergushichev, A. Fast gene set enrichment analysis.

$1013 \quad$ Preprint at bioRxiv https://doi.org/10.1101/060012 (2019). 
1014 95. Liberzon, A. et al. Molecular signatures database (MSigDB) 3.0. Bioinformatics 27, $1015 \quad 1739-1740(2011)$.

1016 96. Andor, N., Harness, J. V., Müller, S., Mewes, H. W. \& Petritsch, C. EXPANDS:

1017 expanding ploidy and allele frequency on nested subpopulations. Bioinformatics 30, 50-

$1018 \quad 60(2014)$

1019 97. Merlo, L. M. F. et al. A comprehensive survey of clonal diversity measures in Barrett's 1020 esophagus as biomarkers of progression to esophageal adenocarcinoma. Cancer Prev. $1021 \quad$ Res. Phila. Pa 3, 1388-1397 (2010).

1022 98. Mroz, E. A. \& Rocco, J. W. MATH, a novel measure of intratumor genetic heterogeneity, 1023 is high in poor-outcome classes of head and neck squamous cell carcinoma. Oral Oncol. $1024 \quad 49,211-215(2013)$.

1025 99. Harrell, F. Regression Modeling Strategies: With Applications to Linear Models, Logistic 1026 Regression, and Survival Analysis. (Springer-Verlag, 2001).

1027 100. Harrell, F. E., Lee, K. L. \& Mark, D. B. Multivariable Prognostic Models: Issues in 1028 Developing Models, Evaluating Assumptions and Adequacy, and Measuring and 1029 Reducing Errors. Stat. Med. 15, 361-387 (1996).

1030 101. Schröder, M. S., Culhane, A. C., Quackenbush, J. \& Haibe-Kains, B. survcomp: an 1031 R/Bioconductor package for performance assessment and comparison of survival models.

1032 Bioinforma. Oxf. Engl. 27, 3206-3208 (2011). 


\section{$\mathrm{a}$}

C Smoker

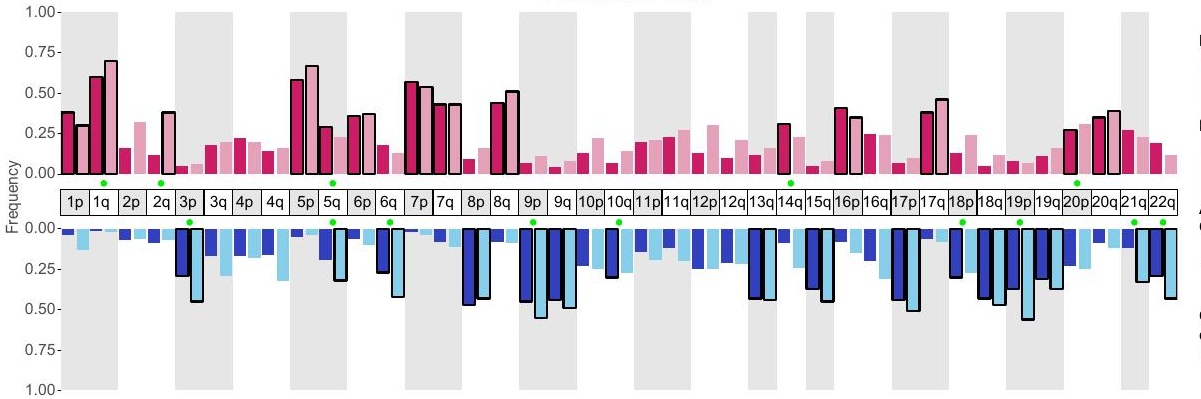

b

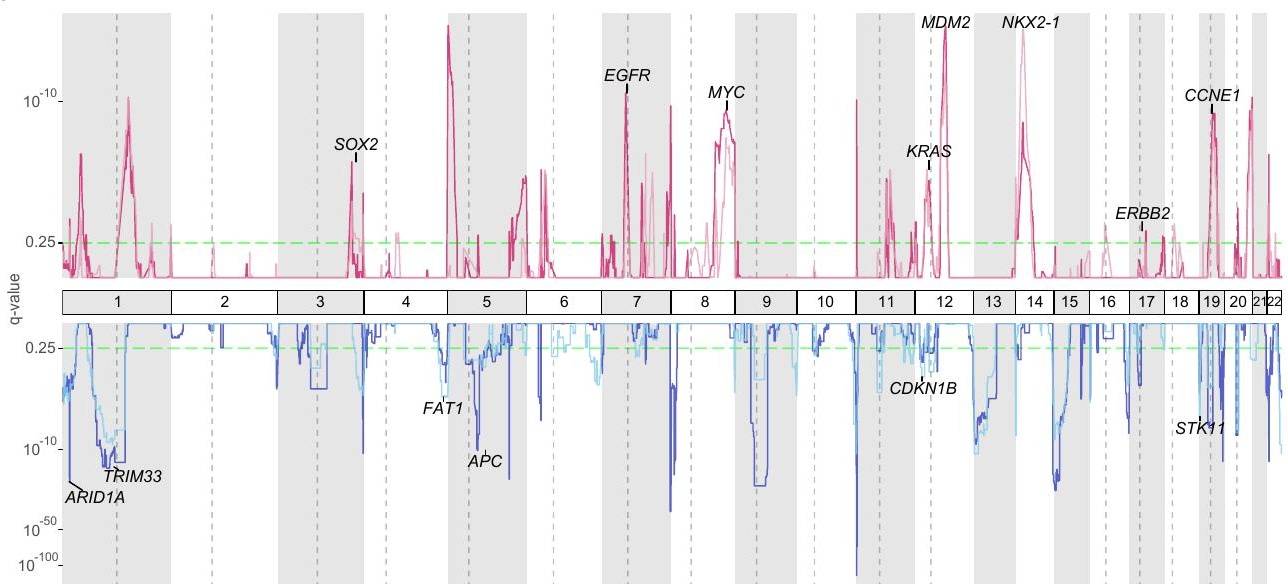

d

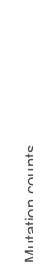

Mutation signature groups

\section{Focal-level CNV}

EAS

Amp Del EUR Amp - Del

Arm-level CNV q-value $\square<0.1$

$\geq 0.1$

Cohort difference
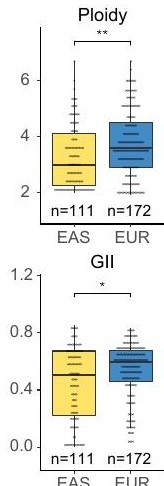

GII.Deletion

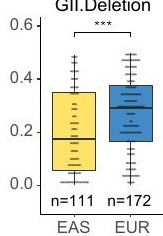

GD

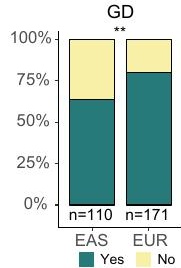

FDR:

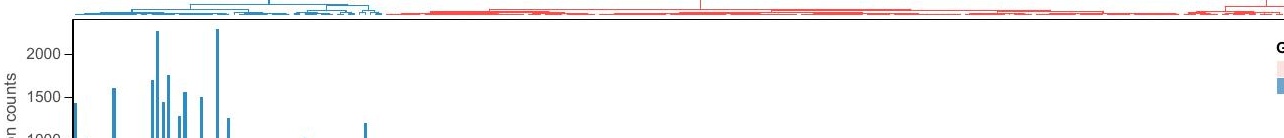

\section{Non-smoker}

Ploidy
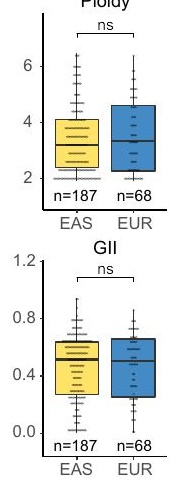

GII.Deletion

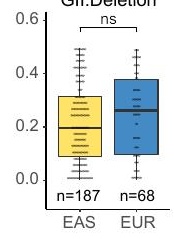

GD

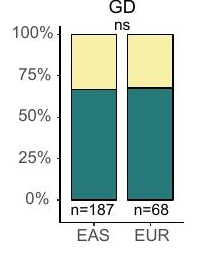

Gender Age Smoker Stage GII EGFR
Female 100 $\begin{array}{llllllr}\text { Female } & 100 & \text { No } & \text { I } & 0.8 & \text { KRAS } \\ \text { Male } & 80 & \text { Yes } & \text { II } & 0.6 & \text { WT }\end{array}$ \begin{tabular}{|ll|l|l|l}
\hline 80 & Yes & II & 0.6 & WT \\
60 & & III & 0.4 & Mut \\
40 & & IV & 0.2 & \\
20 & & & &
\end{tabular}

0 -

G

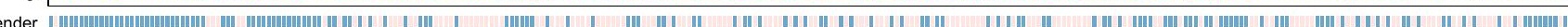

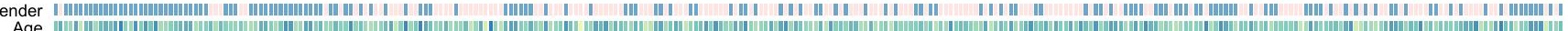

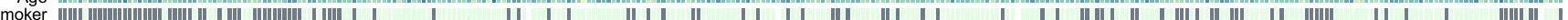

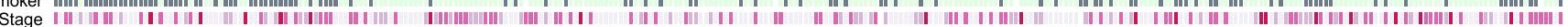

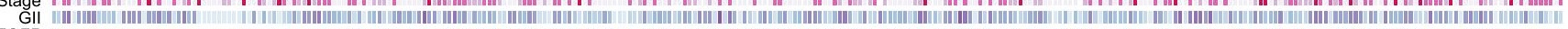

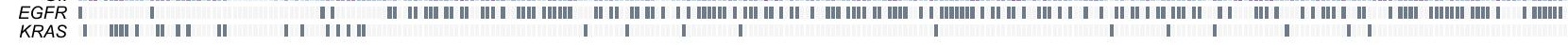




\section{a $\quad$ Smoker}

EAS EUR

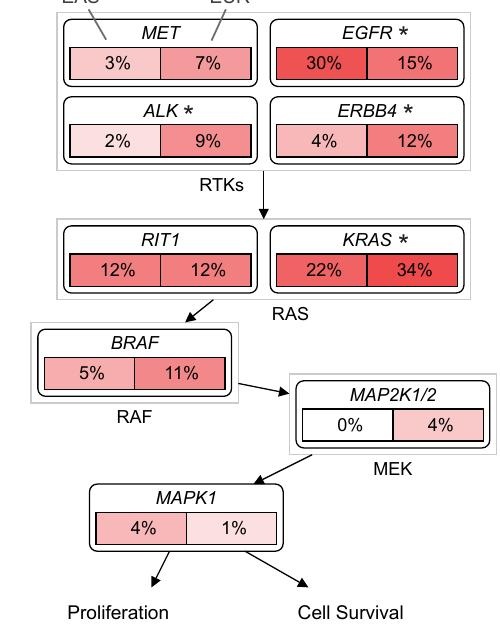

Non-smoker

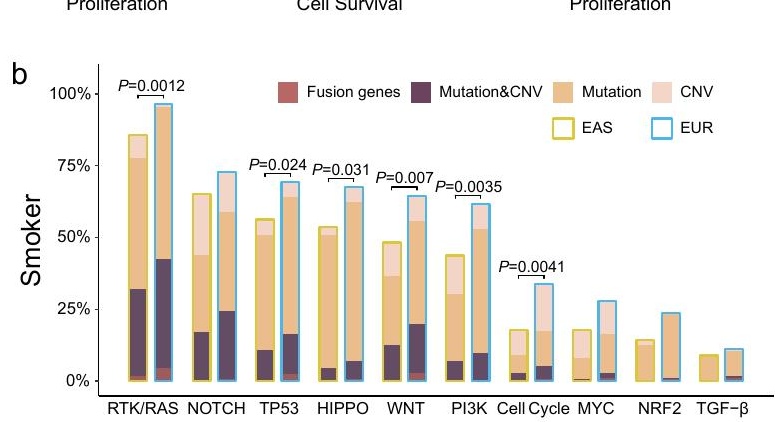

2

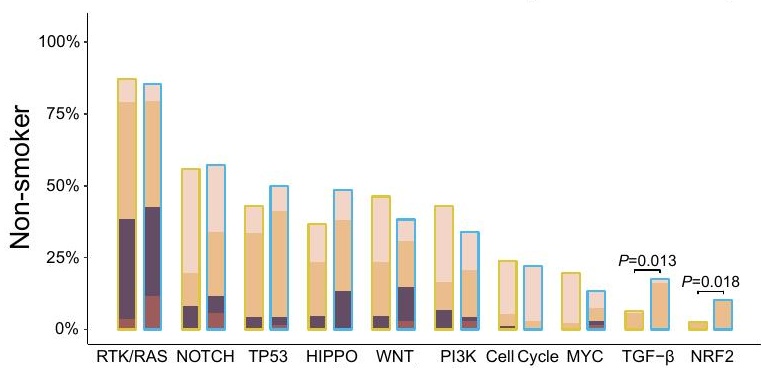
Oncogenic pathways

f

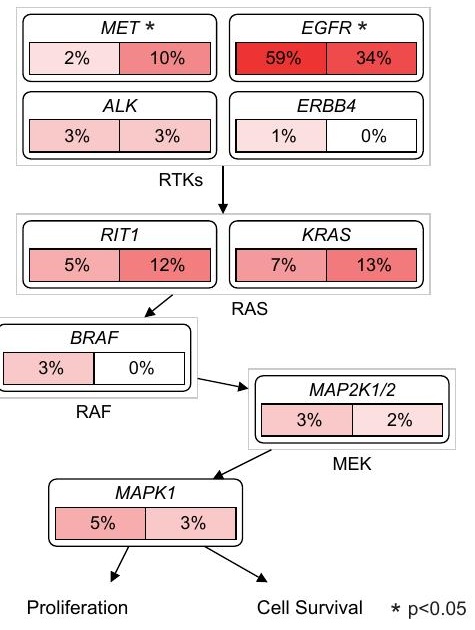

C Smoker

Non-smoker
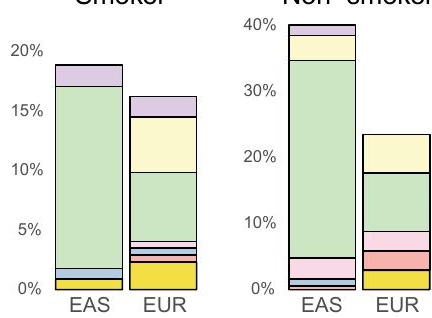

Alteration_OncoKBLv

$\square$ BRAF_Lv1

CDK4.Amp Lv2B

$\square$ EGFR_Lv1

EML4-ALK_Lv1

- ERBB2.Amp_Lv2B

EZR/CD74-ROS1_Lv1

$\square$ MET.Amp_Lv2A

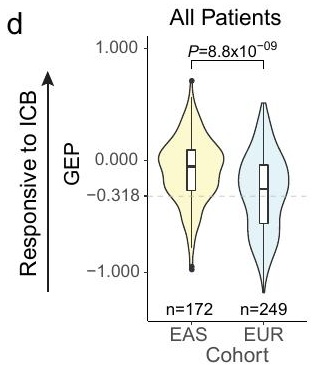

Cohort $\square$ EAS $\square$ EUR
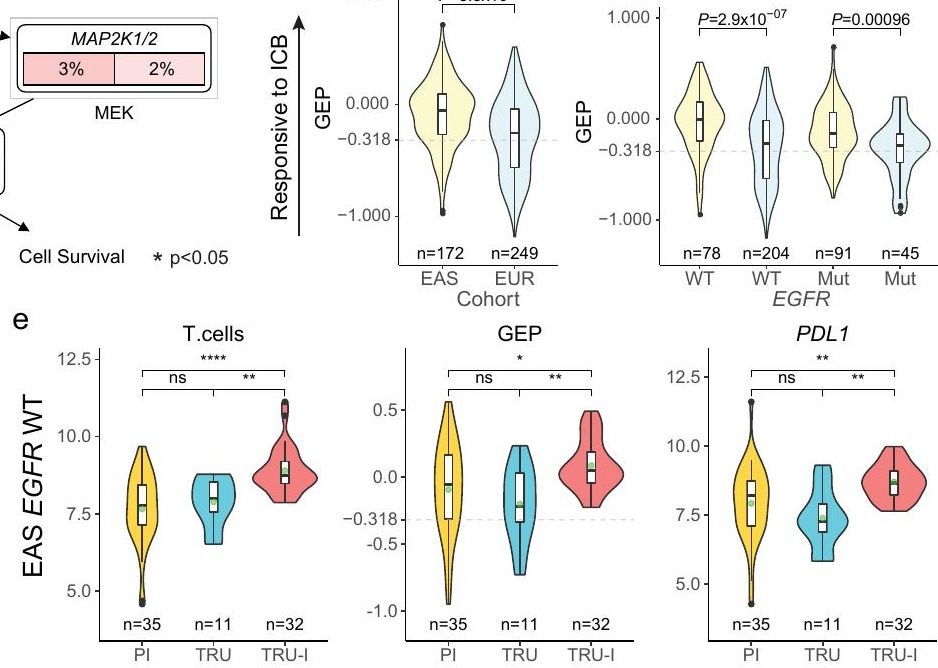

PDL1
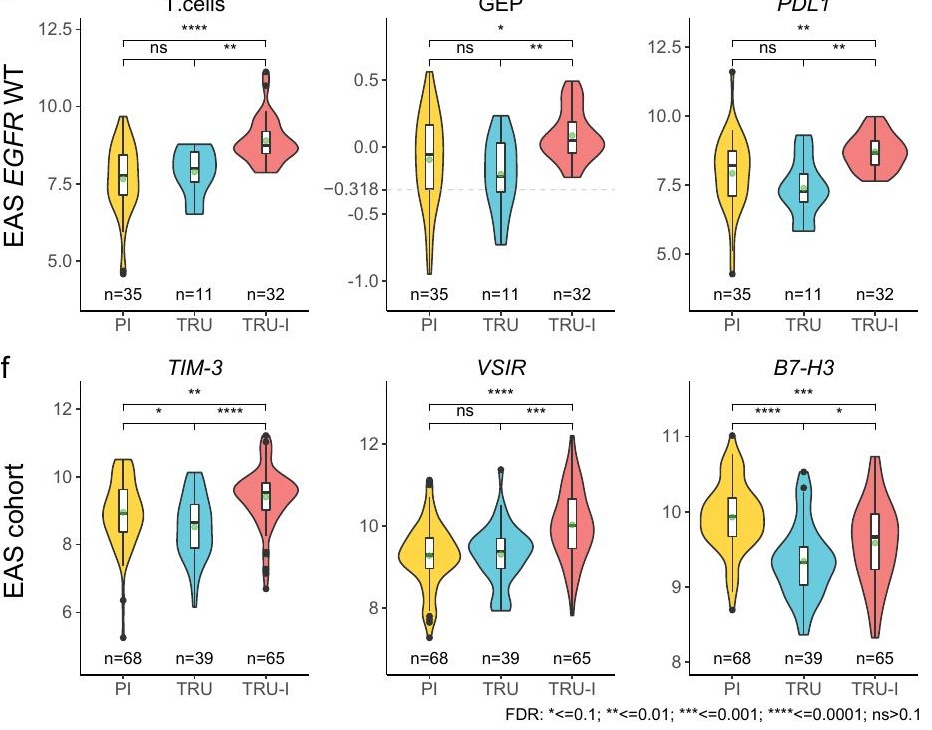












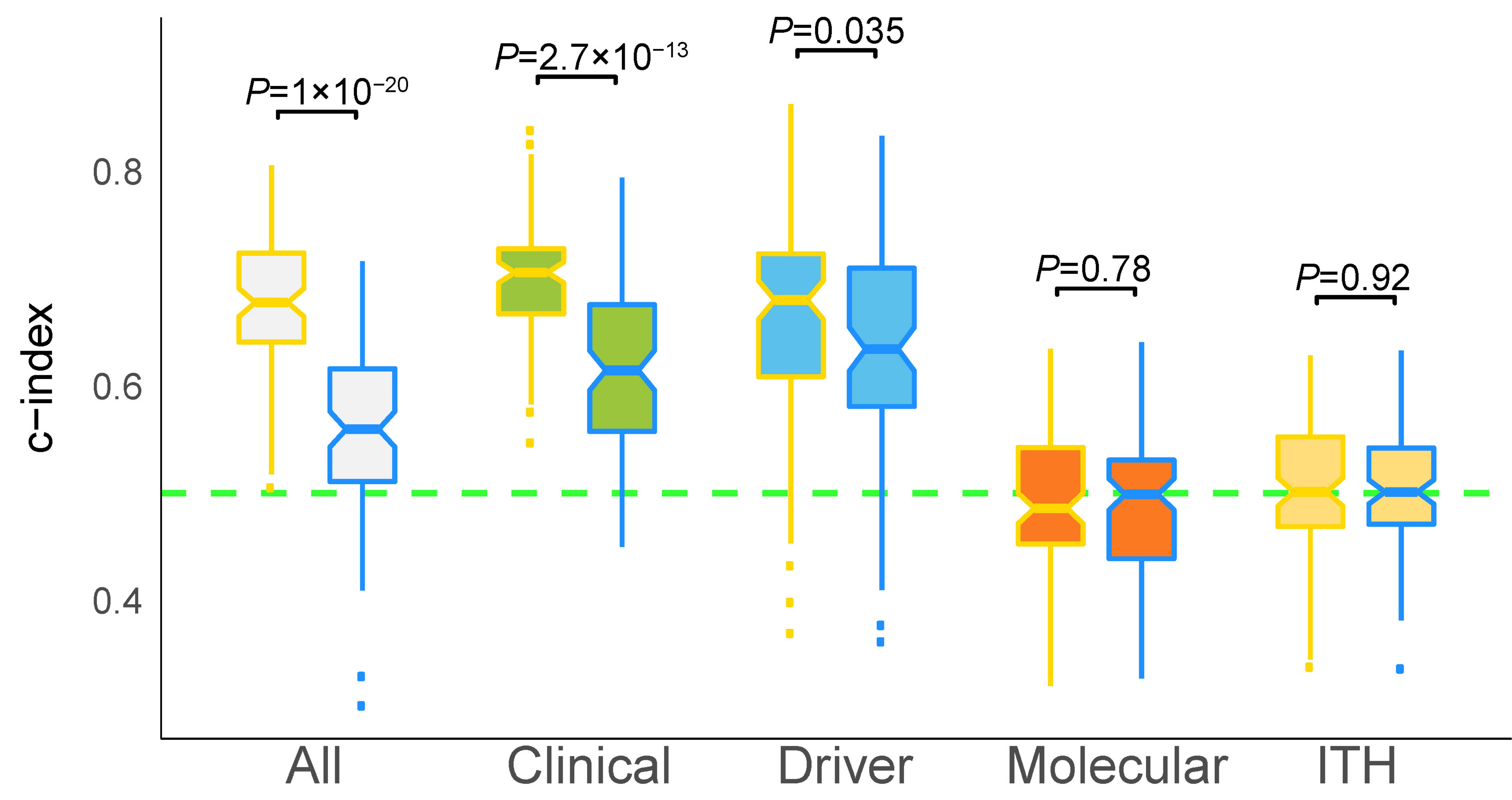

b

EGFR WT, equal proportion of smokers same cohort sizes $(n=112)$

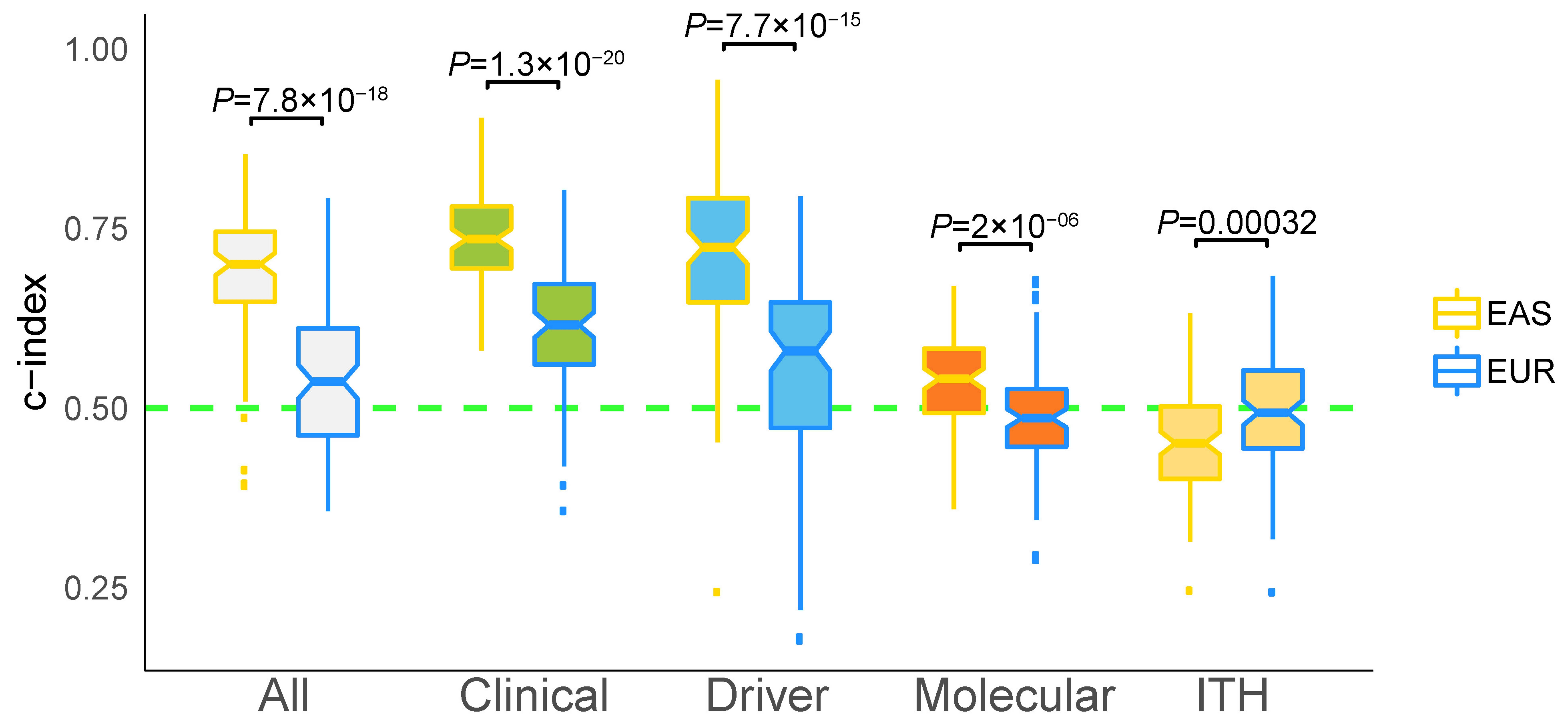




\section{EAS}

EUR

Smoker

EGFR mt.

TMB

GII

RNA-

subtype

TRU-I

PP

IO potential

Prognosis

Accuracy

Smoker

EAS

Gender

Pack year

EGFR mt.

TMB

Num.driver

GII

Ploidy

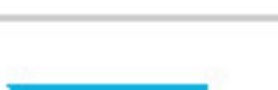

EUR

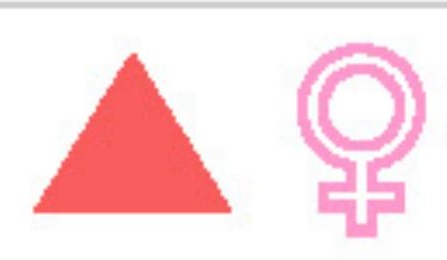

$\Delta$
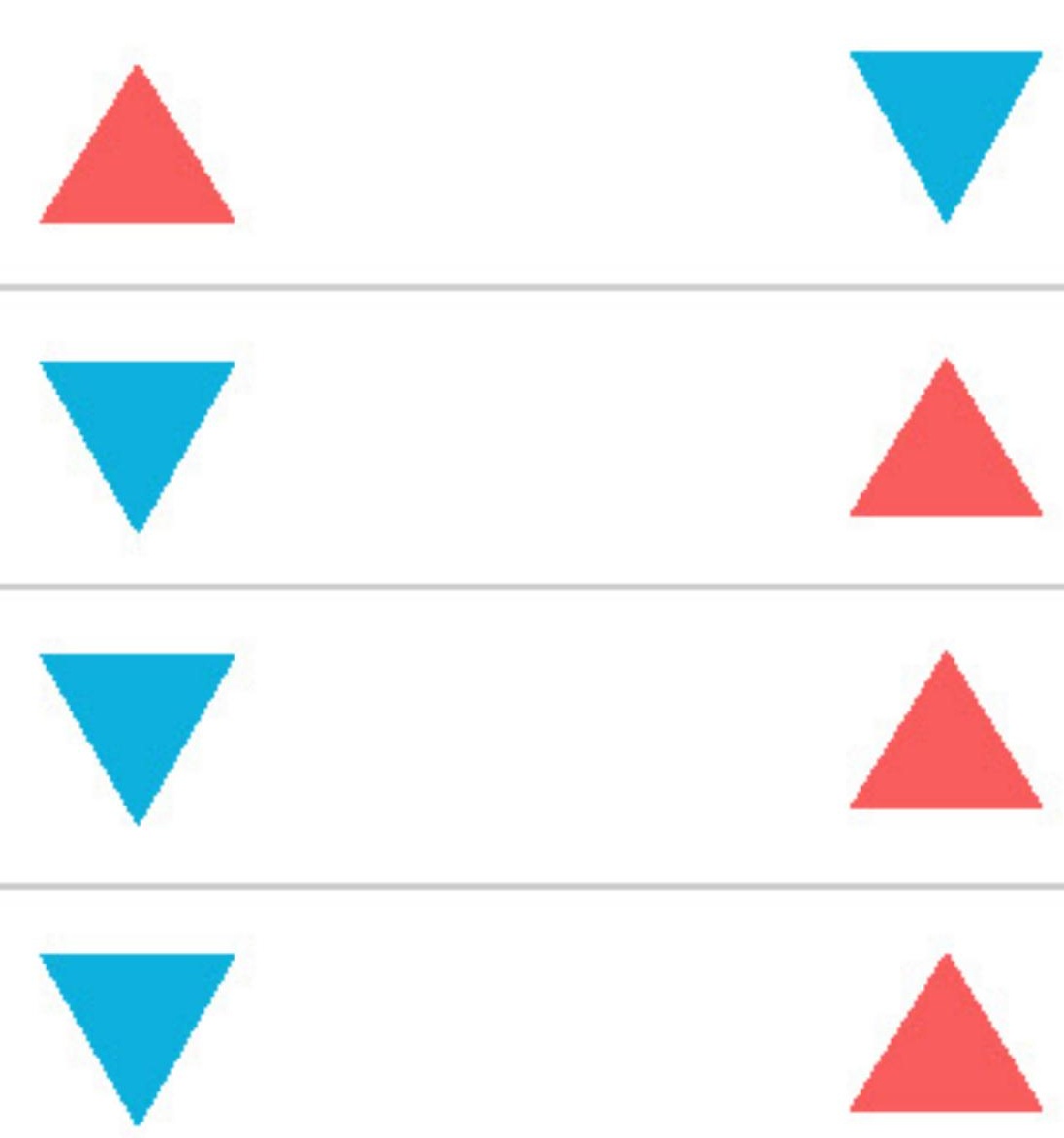

Non-smoker

EAS

EUR
NA

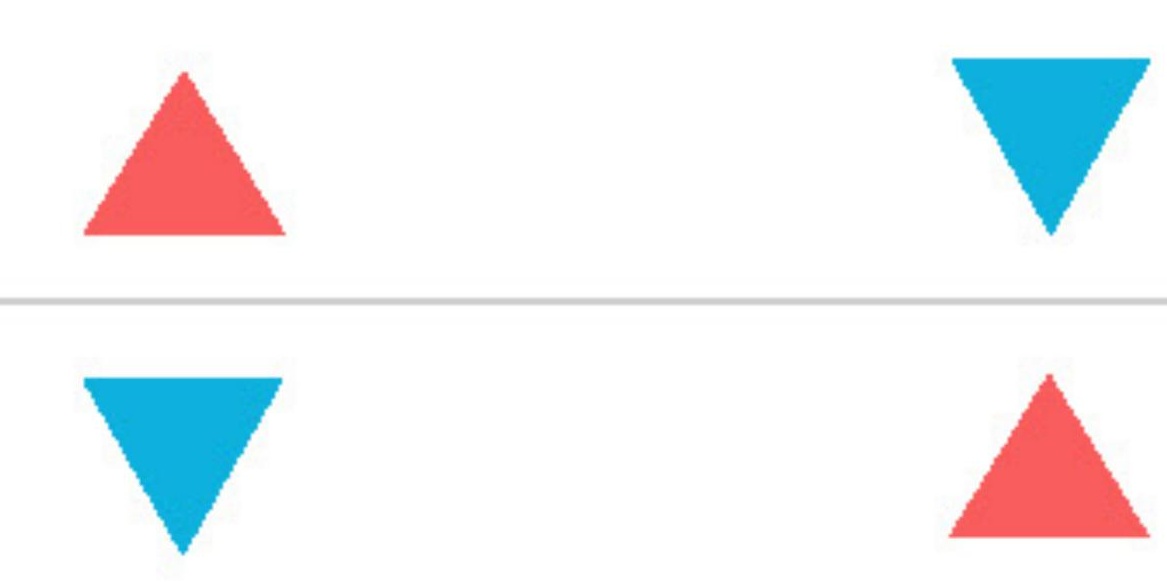

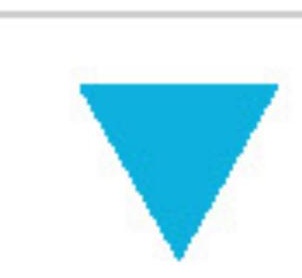

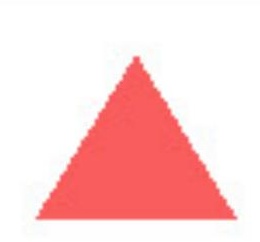

